\title{
Crustal magnetic field of Mars
}

\author{
B. Langlais \\ NAS/NRC at Geodynamics Branch, NASA Goddard Space Flight Center, Greenbelt, Maryland, USA
}

M. E. Purucker

Raytheon ITSS at Geodynamics Branch, NASA Goddard Space Flight Center, Greenbelt, Maryland, USA

M. Mandea

Institut de Physique du Globe, Paris, France

Received 14 January 2003; revised 15 August 2003; accepted 9 September 2003; published 19 February 2004.

[1] The equivalent source dipole technique is used to model the three components of the Martian lithospheric magnetic field. We use magnetic field measurements made on board the Mars Global Surveyor spacecraft. Different input dipole meshes are presented and evaluated. Because there is no global, Earth-like, inducing magnetic field, the magnetization directions are solved for together with the magnetization intensity. A first class of models is computed using either low-altitude or high-altitude measurements, giving some statistical information about the depth of the dipoles. Then, a second class of models is derived on the basis of measurements made between 80 and $430 \mathrm{~km}$ altitude. The 4840 dipoles are placed $20 \mathrm{~km}$ below the surface, with a mean spacing of $2.92^{\circ}$ $(173 \mathrm{~km})$. Residual $\mathrm{rms}$ values between observations and predictions are as low as $15 \mathrm{nT}$ for the total field, with associated correlation coefficient equal to 0.97 . The resulting model is used to predict the magnetic field at $200-\mathrm{km}$ constant altitude. We present the maps of the magnetic field and of the magnetization. Downward continuation of a spherical harmonic model derived from our equivalent source solution suggests that intermediate-scale lithospheric fields at the surface probably exceed $5000 \mathrm{nT}$. Given an assumed $40-\mathrm{km}$-thick magnetized layer, with a mean volume per dipole equal to $3.6 .10^{6} \mathrm{~km}^{3}$, the magnetization components range between $\pm 12 \mathrm{~A} / \mathrm{m}$. We also present apparent correlations between some impact craters ( $\geq 300-\mathrm{km}$ diameter) and magnetization contrasts. Finally, we discuss the implications of the directional information and possible magnetic carriers. INDEX TERMS: 6225 Planetology: Solar System Objects: Mars; 5440 Planetology: Solid Surface Planets: Magnetic fields and magnetism; 5455 Planetology: Solid Surface Planets: Origin and evolution; KEYWORDS: Mars, magnetic field, magnetic anomalies, equivalent sources, magnetization

Citation: Langlais, B., M. E. Purucker, and M. Mandea (2004), Crustal magnetic field of Mars, J. Geophys. Res., 109, E02008, doi:10.1029/2003JE002048.

\section{Introduction}

[2] Prior to the launch of Mars Global Surveyor (MGS) in 1996, the magnetic field of Mars was poorly known, and its origin was controversial. Previous spacecraft missions only gave an upper limit on the magnetic moment of the planet. Trotignon et al. [1993] gave a value of $2.10^{12} \mathrm{~T} . \mathrm{m}^{3}$, to be compared to the Earth's magnetic moment of $8.10^{15}$ T.m ${ }^{3}$. The low altitude magnetic measurements of MGS were thus eagerly awaited.

[3] MGS carries two triaxial fluxgate magnetometers, identical to the magnetic field experiment on board the Mars Observer mission [Acuña et al., 1992]. This configuration provides a way to deduce and remove the spacecraft-generated magnetic fields. The field measurements

Copyright 2004 by the American Geophysical Union. 0148-0227/04/2003JE002048 used in this study have had static and dynamic spacecraft fields removed [Acuña et al., 2001].

[4] The main objective of the MGS magnetic experiment was to determine the nature of the magnetic field of Mars. This goal requires models and maps to be computed, with resolution in accordance with the satellite altitude and orbit. First attempts to describe and interpret Mars' magnetic field [Acuña et al., 1999; Connerney et al., 1999] were limited because of the wide range of the measurement altitude (from 90 to $200 \mathrm{~km}$ above the reference radius $3393.5 \mathrm{~km}$ ). The altitude is indeed a key factor in determining the intensity of the magnetization, since the magnetic sources are close to the surface [Stevenson, 2001]. It is thus necessary to have maps of magnetic components (measurements or predicted by a model) at a constant altitude over the sphere, so that the anomalies can be laterally characterized. It is also crucial to be able to downward or upward continue such maps. Having multiple 
altitude coverage greatly helps to characterize the properties of the magnetized bodies.

[5] More recent attempts to model the magnetic field of Mars were done, using different techniques and data sets. Purucker et al. [2000] used low altitude, purely radial, preliminary binned, MGS magnetic observations to produce a constant altitude map of the magnetic field, using an equivalent source approach. Other studies dealt with the commonly used (for the Earth's magnetic field) spherical harmonic method [Gauss, 1839]. Arkani-Hamed [2001a] used three components of the same low-altitude observations to produce a spherical harmonic model up to degree and order 50. This model was later updated [Arkani-Hamed, 2002], using both low- and high-altitude measurements. Using the same kind of data, Cain et al. [2003] derived another spherical harmonic model, but up to degree and order 90 .

[6] In this study, we extend the work of Purucker et al. [2000], by introducing the three components of both the magnetic observations and those of the equivalent sources. When dealing with the Earth's magnetic field, spherical harmonic analysis is usually the technique of choice for representing the large core field, while equivalent source dipoles are largely used for lithospheric field representations [Langel and Hinze, 1998]. There are several reasons for this. First, spherical harmonic analysis is computationally more demanding for the high degree solutions needed to fully represent the lithospheric field, even if models based on satellite data (MAGSAT, Ørsted and CHAMP) take into account the lithospheric field [Cain et al., 1989; Langlais et al., 2003; Maus et al., 2002]. Second, the MGS magnetic measurements were acquired between 80 and $450 \mathrm{~km}$ altitude, with an uneven data geographical distribution and with an estimated accuracy of 3 nT [Acuña et al., 1998]. Spherical harmonic analysis is efficient, provided there exists an almost constant geographical coverage, with a reasonable data accuracy. Following Schmitz et al. [1989], noise associated with data acquired at or near a $400-\mathrm{km}$ spherical shell, on a $2^{\circ}$ or $3^{\circ}$ side equiangular grid, must be lower than $2 \mathrm{nT}$ to compute Gauss coefficients that are be reliable up to degree 44 (even down to $30-35$ in the conservative case). In contrast, the equivalent source dipoles approach is less sensitive to geographical data distribution. Furthermore it is capable of providing insight into the magnetization directions in the source region.

[7] The main objective of this study is to provide a new model of the Martian magnetic field. This model is designed to be used for predictions of the three components of the Martian magnetic field at altitudes ranging between $173 \mathrm{~km}$, the mean horizontal resolution of our model, and $430 \mathrm{~km}$, the maximum altitude of the MGS measurements we used in our model. This new discrete magnetization model is the first global model that can explain the magnetic field measurements in terms of possible lithospheric sources, despite the non-uniqueness of the solution; one can in particular add any magnetization distribution that does not produce a magnetic field outside the source region [Runcorn, 1975]. Indeed, other models are either description of the field expressed on a spherical harmonic basis [Arkani-Hamed, 2002; Cain et al., 2003] or incomplete and physically meaningless equivalent source approaches (Purucker et al. [2000] used only radial dipoles to produce a constant altitude map of the radial magnetic field).
[8] In the following we review our basic modeling methods. We then introduce the low-altitude and highaltitude data sets, which are used to produce independent models, and compared for consistency. We then compute a global magnetization model. We predict the magnetic field at a constant altitude of $200 \mathrm{~km}$ and discuss its morphology. We then extend the discussion to some particular outputs of our model, such as the relatively weak magnetization over large craters ( $\geq 300 \mathrm{~km}$ diameter), and the possible magnetic carriers.

\section{Data}

[9] A review of MGS design and science objectives can be found in Albee et al. [2001]. We therefore briefly recall what is relevant here. MGS was launched on November 7th, 1996. It reached Mars' environment and was inserted into orbit on September 11th, 1997. Three phases were initially planned. The first phase was AeroBraking (AB), during which the satellite was slowed and the orbit evolved from highly elliptical to almost circular. After a six-month $\mathrm{AB}$ phase the Mapping Orbit (MO) was scheduled to begin. However, because a problem occurred during the deployment of one of the solar panels, the circularization of the orbit had to be slowed [Albee et al., 1998]. The AB phase was split into two distinct phases (AB1 and AB2, lasting 7 and 5 months each, respectively), separated by a six-month interval (in order to allow the orbit to drift into the proper position with respect to the Sun), during which scientific instruments were turned on. This phase was called the Science Phasing Orbit (SPO). The orbit during this SPO phase was elliptical, with periapsis as low as $80 \mathrm{~km}$ with respect to the reference radius of $3393.5 \mathrm{~km}$. Following the AB2 phase, MGS entered the MO phase, where it has been since March 1999. The final orbit is a $400-\mathrm{km}$, near-circular orbit.

[10] In this study, we use the magnetic measurements made available by the MAGnetometer/Electron Reflectometer (MAG-ER) team, and distributed by the Planetary Plasma Interactions Node of the UCLA Planetary Data System (PDS). Magnetic data were expressed in a Cartesian, planetocentric system and in a Cartesian, Sun-related system. We used the first system to compute the position of the satellite and the magnetic components in the spherical planetocentric system, with radius $r$, colatitude $\theta$ and longitude $\phi$. The second system was used to determine whether the satellite is in the sunlit or shadow side of Mars, supplemented with solar panels outputs made available as part of the PDS set. Measurements used in this study were acquired during the AB1, SPO, AB2 and MO (during 1999) phases. We limited the altitude of the $\mathrm{AB}$ and $\mathrm{SPO}$ measurements to $350 \mathrm{~km}$, while the one for the MO measurements was imposed by the orbital parameters, between 360 and $440 \mathrm{~km}$, with a periapsis always located near the South Pole.

[11] The AB, SPO and MO data sets can be considered as providing dual coverage of the Martian surface, one near $400 \pm 30 \mathrm{~km}$ (MO data set), and the second near $200 \pm$ $100 \mathrm{~km}$ (AB and SPO data sets). Unfortunately, the lowest coverage is far from being complete. $62 \%$ of the $1^{\circ} \times 1^{\circ}$ bins below $300 \mathrm{~km}$ are filled, and only $48 \%$ of the bins below $200 \mathrm{~km}$ are. Moreover, only 50\% and 37\%, respectively, of those bins contain more than 2 observations. 
[12] The two raw data sets were processed to yield two equiangular data sets. First, a night local time selection was applied for the SPO and MO data sets in order to reduce the external magnetic perturbations. Because of the orbital parameters during the $\mathrm{AB}$ phases, $80 \%$ of the data were from the dayside, and we chose to keep them. Second, we looked for possible outliers in the measurements. These outliers were identified by comparing the vertical magnetic field measurements with those predicted by an earlier model derived by Purucker et al. [2000]. All measurements with associated absolute residuals larger than $50 \mathrm{nT}$ (for the $\mathrm{AB}$ and SPO data sets) were removed, corresponding to $0.1 \%$ out of the $2.7 \times 10^{6}$ measurements. MO measurements associated with residual magnitudes larger than $9 \mathrm{nT}$ were removed, corresponding to $12 \%$ out of $8.4 \times 10^{6}$ measurements. However, because of their dense geographical distribution, the density of the final high-altitude data set was not altered, although the number of measurements per bins changed. Finally, we computed mean magnetic values in each $1^{\circ} \times 1^{\circ} \times 10 \mathrm{~km}$ cell, obtaining 75380 binned values for the MO data set, and 103996 for the AB and SPO data sets (below $350 \mathrm{~km}$ altitude). When more than 2 measurements were in a cell, an associated variance (with respect to the mean value) was computed. For more than $50 \%$ of the low-altitude cells, it was not possible to compute such a variance.

\section{Modeling}

[13] The approach adopted here is the equivalent source dipole technique, introduced by Mayhew [1979] for the representation of satellite magnetic field data. Using as input irregular and scattered magnetic measurements acquired on local or global scales, we can use equivalent dipoles to predict the magnetic measurements in a leastsquares fit. Considering the magnetic moment $M$ of a dipole located at $\left(r_{d}, \theta_{d}, \phi_{d}\right)$, the magnetic potential observed at $(r, \theta, \phi)$ is expressed as

$$
V=-M \cdot \nabla \frac{1}{l}
$$

This relation is valid provided that there are no sources between the dipole and the observation location. The distance $l$ between the dipole and the observation location is written

$$
l=\left(r_{d}^{2}+r^{2}-2 r_{d} r \cos (\zeta)\right)^{\frac{1}{2}}
$$

$\zeta$ being the angle between observation and dipole location:

$$
\cos (\zeta)=\cos (\theta) \cos \left(\theta_{d}\right)+\sin (\theta) \sin \left(\theta_{d}\right) \cos \left(\phi-\phi_{d}\right)
$$

[14] The resulting magnetic field $\vec{B}$ is written as

$$
\vec{B}=-\vec{\nabla} V=-\left(\frac{\partial}{\partial r}, \frac{\partial}{r \partial \theta}, \frac{\partial}{r \sin (\theta) \partial \phi}\right) V
$$

[15] On the Earth, we generally suppose that the magnetization is aligned along the direction of the main field [Purucker et al., 1996]. In this case, one looks only for the dipole moment $M$ of the anomaly, its three components are written as $(M \sin I, M \cos I \cos D, M \cos I \sin D), I$ and $D$ being the inclination and the declination of the main magnetic field. In the case of Mars, there is no main field of core origin, hence no organizing magnetic field for purposes of induction. Thus $I$ and $D$ can be considered laterally uncorrelated on large length-scales.

[16] The only previous similar Martian study was made using the vertical component of the low-altitude, uncalibrated and pre-processed, magnetic field measurements, and assumed vertical magnetizations [Purucker et al., 2000]. The present study differs from that one as we considered the three components $\left(M_{r}, M_{\theta}, M_{\phi}\right)$ of the magnetization, constrained by using all three components $\left(B_{r}, B_{\theta}, B_{\phi}\right)$ of the magnetic field measurements acquired at both low- and high-altitude. We used a least-square approach, by minimizing the weighted root mean square difference between measurements and predictions by the model. The weights we used are the variances computed for each $1^{\circ} \times 1^{\circ} \times$ $10 \mathrm{~km}$ cell. We used a conjugate gradient iterative technique to solve for the system. The complete expressions are given in Appendix A.

[17] The magnetic anomaly as measured at one place is the sum of the magnetic anomalies created by all dipoles, but only those within a certain range contribute significantly. Numerous tests performed by Purucker et al. [1996] showed that this range can be confined to a spherical volume of radius $1500 \mathrm{~km}$. Although not used in this study, another obvious advantage of this approach compared to spherical harmonic analysis is that we can consider either the global problem or a smaller area of the planet, using the same method.

\section{Input Dipole Meshes}

[18] As the problem has a non-unique solution, one needs to carefully select the modeling parameters. These can be classified in two groups. The first group is the location of the dipoles, whereas the second one is the magnetization intensity and direction. The dipole locations are typically placed a priori, as the non-linearity makes it difficult to solve for all parameters at the same time.

[19] The dipole geographical distribution should be as homogeneous as possible in order to minimize the sources of instabilities [Covington, 1993], assuming the data distribution is homogeneous (which is the case in this study). Here an icosahedral discretization of the sphere [Vestine et al., 1963] was chosen. To obtain such a distribution, one first needs to project on the sphere twelve vertices: one at the North Pole, five equiangular distributed points at $30^{\circ} \mathrm{N}$ latitude, five others at $30^{\circ} \mathrm{S}$ latitude, and one at the South Pole. These twelve points define a mesh of 20 equal spherical triangles, bounded by thirty geodesic arcs. One then can easily increase the discretization, by connecting equi-distance points on the geodesic arcs, thus resulting in smaller spherical triangles.

[20] In our computations, we used different levels of refinement, by changing $i_{s}$, the number of points per arc (there are $i_{s}-1$ divisions per arc). In the following we will refer to $i_{s}$ as the dipole parameter. The relationship between $i_{s}$ and $m$, the number of dipoles located at the nodes of the spherical triangles, is

$$
m=10 \times\left(i_{s}-1\right)^{2}+2
$$


[21] For example, $i_{s}=20$ results in 3612 dipoles, of which we retained only 3610 within the $\pm 88^{\circ}$ latitude band (in order to reflect the orbit inclination of MGS). To compute the mean spacing, we divided the spherical surface area by the number of dipoles, the square root of this value (the mean surface) indicating the mean spacing, in this case $3.38^{\circ}$.

[22] We assumed the magnetization to be confined to a $40-\mathrm{km}$ thick layer, consistent with previous studies. This magnetic thickness is comparable to the inferred mean crustal thickness of $\sim 50 \mathrm{~km}$ [Zuber, 2001; Smith and Zuber, 2002]. A few previous studies dealt with estimates of magnetic thickness. On the basis of the interpretation of the energy spectrum of the Martian magnetic field, Voorhies et al. [2002] give a value less than or equal to $50 \mathrm{~km}$ for the magnetic thickness. By comparing magnetic measurements made above the largest impact craters of Mars, Nimmo and Gilmore [2001] give a mean magnetic thickness value of $35 \mathrm{~km}$. The thickness, however, is not a crucial parameter, as we have only access to the vertically integrated magnetization. The predicted magnetic field will be slightly affected by this thickness. Instead, we varied $h$ the depth to the top of this layer, from $+10 \mathrm{~km}$ (above the mean $3393.5-\mathrm{km}$-radius sphere) to $-40 \mathrm{~km}$ (below). Although having sources above the surface is not realistic, this choice allows us to estimate the importance of this parameter with regards to the fit to the data.

\section{Modeling Results}

[23] We computed magnetization models, using different depths for the dipoles, and different resolutions for the dipole meshes. In the following, models are denoted as $\mathrm{M}\left[i_{s}\right] /[h] /[k], k$ being the $k$ th iteration. M23/+00/10 then refers to a dipole mesh with $i_{s}=23$ (mean spacing $=2.72^{\circ}$ ), located at the surface $(+00 \mathrm{~km})$, from which we retained the 10 th iteration as the final solution. In each case, the inversion was stopped after 100 iterations or when the weighted residual rms values calculated after each iteration did not decrease by more than $10^{-5}$ (whichever came first).

[24] We then predict the magnetic observation using each iteration of each solution. Because of the non-uniqueness of the solution, we tried to develop some objective criteria to decide which solution should be considered the most reliable. The criteria we chose are based on the evolution of the standard deviation and correlation coefficients between observed and predicted magnetic measurements with respect to the evolution of the extrema and root mean square values of the magnetization components. We computed residual rms values $\sigma_{C}$ between observed and predicted field components $C$, expressed as

$$
\sigma_{C}=\left[\frac{\sum_{i=1}^{n}\left(C_{\mathrm{obs}}-C_{\mathrm{mod}}\right)^{2}}{n}\right]^{1 / 2}
$$

where $C_{\mathrm{obs}}$ and $C_{\mathrm{mod}}$ refer to the observed and predicted values, respectively. The choice of this criteria is discussed later.

[25] In the following, we first present magnetization models derived from partial, altitude-dependent, data sets. Indeed a very simple way to test our modeling assumptions together with the magnetic measurements is to derive magnetization models based on a fraction of the full data set, and to use these resulting models to predict the unused data. Then we introduce the global magnetization model.

\subsection{Solutions Based on Partial Data Sets}

[26] Using either the low-altitude, AB and SPO data sets, or the high-altitude, $\mathrm{MO}$ data set, we computed magnetization models using different input dipole mesh resolutions and depths. These models (for all mesh resolutions, depths and iterations) were then used to predict either the highaltitude, MO data set, or the low-altitude, AB and SPO data sets. In the following, these tests are referred as high-to-low (MO-based models, AB and SPO predictions), high-to-high (MO-based models, MO predictions), low-to-high (AB-, SPO-based models, MO predictions), and low-to-low (AB-, SPO-based models, AB and SPO predictions). Input dipole meshes had parameters $i_{s}$ ranging from 12 to 30 (or from 5.83 to $2.21^{\circ}$ ), and dipole depths $h$ from -40 to $+10 \mathrm{~km}$, with a $10-\mathrm{km}$ increment.

[27] Let us first consider the low-to-low and low-to-high cases. Models are based on 49241 binned measurements, located between 80 - and $300-\mathrm{km}$ altitude, with an uneven geographical data coverage. There is a very fast convergence between MO observations and MO predictions, followed by a divergence starting after the 5th or the 6th iteration independent of the depth or the mesh resolution.

[28] - We analyzed the evolution of the residual rms values for constant mesh resolution $\left(i_{s}=23\right)$, with varying dipole depth. The low-to-low rms values are minimum for depths of $-20 \mathrm{~km}$; total field residuals are then $23.98 \mathrm{nT}$. Corresponding low-to-high residual rms values increase slightly as the dipoles are put deeper (from 4.96 to $5.32 \mathrm{nT}$, but the major increase occurs between -20 - and $-30-\mathrm{km}$ depths, and between -30 - and $-40-\mathrm{km}$ depths). At the same time, low-to-high and low-to-low correlation coefficients are almost constant.

[29] $\bullet$ We then analyzed the evolution of the rms values for constant depth $(-20 \mathrm{~km})$, and varying dipole mesh parameter. The low-to-high total field rms values are almost constant (between 5.04 and $5.35 \mathrm{nT}$ for total field residuals), but a minimum is observed for $i_{s}=16$ (mean spacing $4.28^{\circ}$ ). Corresponding low-to-low total field residual rms values decrease as the dipole mesh parameter increases. However, this evolution is less significant for $i_{s} \geq 23$, when rms values are then $\simeq 23-24 \mathrm{nT}$.

[30] Let us now turn to the high-to-low and high-to-high cases. A total of 74446 bins, from $350-$ to $450-\mathrm{km}$ altitude, with an almost homogeneous geographical data distribution, were used. Again, the residuals start to increase after a number of iterations. The deeper the dipoles are, or the finer the dipole mesh is, the later the divergence begins.

[31] - We analyzed the evolution of the residual $\mathrm{rms}$ values when considering a constant dipole mesh parameter $\left(i_{s}=23\right)$. The minimum high-to-high total field residuals are observed for depths $h$ equal to -20 and $-30 \mathrm{~km}$. The highto-low total field residuals decrease as the dipoles are deeper. However, the evolution of the residual rms values is much more significant above $-10 \mathrm{~km}$ than below $-20 \mathrm{~km}$.

[32] - We then analyzed the residual $\mathrm{rms}$ values for a constant depth of $-20 \mathrm{~km}$. The minimum $(3.7 \mathrm{nT})$ high-tohigh total field rms values are reached for $i_{s}$ equal to 25 (mean spacing $2.68^{\circ}$ ). Following that minimum, almost null 

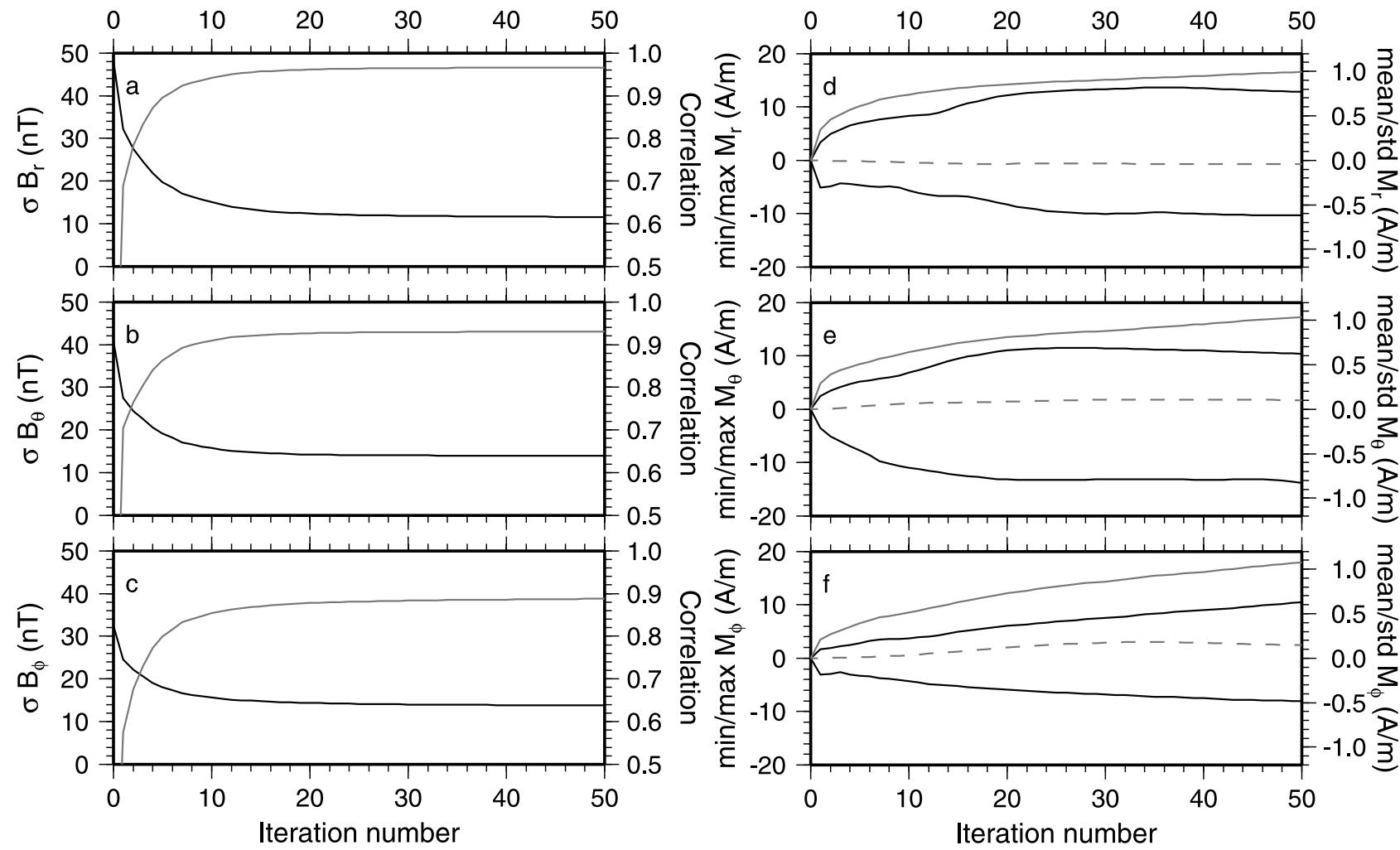

Figure 1. Statistics of the M22/-10 model series. Left Panel: evolution of rms (black line: left axis) and correlation coefficient (gray line: right axis) between observed and predicted values for a) $B_{r}$, b) $B_{\theta}$ and c) $B_{\phi}$. Right Panel: minimum and maximum (black solid line: left axis), arithmetic mean (gray dashed line: right axis) and std mean magnetizations (gray solid line: right axis) for d) $M_{r}$, e) $M_{\theta}$ and f) $M_{\phi}$.

divergences between AB-, SPO-measurements and AB-, SPO-predictions are observed. The high-to-low rms values do not show a minimum for increasing $i_{s}$, although their evolution is very low for $i_{s}$ larger than $27\left(2.46^{\circ}\right)$.

[33] These tests first show the good correlation between the high- and low-altitude data sets and confirm the lithospheric origin of the Martian magnetic field. These tests also give some constraints on the mean, statistical depth to the sources. In both the high-to-high and low-to-low cases, minimum residual rms values are observed for a depth $h$ equal to $-20 \mathrm{~km}$. The residuals computed for the highto-high case do not decrease for large $i_{s}$. On the contrary, the low-to-low residual rms values (correlation coefficients) slightly decrease (increase) for increasing $i_{s}$.

[34] This can be interpreted in terms of optimal dipole mesh resolution. The models based on the low-altitude, AB and SPO data sets, could be under-parameterized, while those based on the high-altitude, MO data set, might be over-parameterized. This result may, however, be biased by the uneven data distribution at low altitudes, or by the nonmodeled external magnetic field. However, the magnetization models based on these partial, low- or high-altitude data sets, are able to correctly predict the unused data. It is thus possible to combine these low- and high-altitude data sets in an unique problem, in order to get a more complete description of the magnetization distribution.

\subsection{Global Solutions}

[35] We also computed magnetization models based on the full data set. Input dipole depths were identical to those of the partial solutions. We restricted the dipole mesh parameter $i_{s}$ to between 12 and 23, because of the amount of memory required. Then we computed the residual rms values and the correlation coefficients, and we used these values to decide which iteration we should retain for each solution.

[36] Figures 1a-1c show an example of the evolution of these statistics for the M22/-10 series. The residual rms values decrease very fast, reaching an almost constant value after the 25th iteration or so. The correlation coefficients increase similarly. It is thus difficult to pick a solution, especially when considering the statistics of the solution (Figures 1d-1f): for almost identical rms, the magnetization can change dramatically, without creating any coherent magnetic field at the satellite altitude. This can be seen as an annihilator of the system [Parker, 1977].

[37] Here we define an objective, automatic criterion to choose a solution. Among the possible criteria, we used the relative change between consecutive iterations of the residual rms values. This corresponds to computing the slope of the evolution of the rms for each iteration. Several limits were tested, including 5.0, 1.0, 0.5, and $0.1 \%$. For the M22/ -10 series, this corresponds to the 6 th, 13 th, 18 th and 25 th iteration, respectively. Detailed statistics of this series are given in Table 1. While the residuals between observed and predicted vertical components decrease slowly (by $8.0 \%$ and $15.3 \%$ between the 13th and the 18th iteration and between the 13 th and the 51 st iteration, respectively), the rms mean vertical magnetization increases significantly (by more than $6.6 \%$ and $26.6 \%$, respectively). This rms magnetization increase is even more drastic for the North-South $(11.2 \%$ 
Table 1. Residual rms Values and Magnetization Statistics for M22/-10 Series

\begin{tabular}{|c|c|c|c|c|c|c|c|c|c|c|}
\hline \multirow[b]{2}{*}{ Limit } & \multirow[b]{2}{*}{ Iteration } & \multicolumn{3}{|c|}{ Rms Res. (nT) } & \multicolumn{3}{|c|}{ Correlation } & \multicolumn{3}{|c|}{ Rms Mag. $(\mathrm{A} / \mathrm{m})$} \\
\hline & & $B_{r}$ & $B_{\theta}$ & $B_{\phi}$ & $B_{r}$ & $B_{\theta}$ & $B_{\phi}$ & $M_{r}$ & $M_{\theta}$ & $M_{\phi}$ \\
\hline $0^{0}$ & 06 & 185 & 187 & 173 & 0.909 & 0878 & 0817 & 0,64 & 0.53 & \\
\hline & & & & & & & & & & \\
\hline & & & & & & & & & & \\
\hline & 2 & & & & & & & & & \\
\hline $0 \%$ & 51 & 11.6 & 14.0 & 13.8 & 0.966 & 0.930 & 0.888 & 0.995 & 1.041 & 1.07 \\
\hline
\end{tabular}

and $47.9 \%$ ) and East-West (18.8\% and 86\%) components, while the residual rms values do not decrease by more than $2-3$ and $6-8 \%$, respectively. This behavior is also observed for different dipole parameters and depths. The increase of the magnetization values, not associated with the increase of the magnetic field at the satellite altitude, could be linked to some leakage of an annihilator in the magnetization distribution. It appears that the $1 \%$ limit applied to the relative evolution of the residuals is a good choice. The resulting model provides a good fit to the data, without being too energetic in amplitude.

[38] Figure 2 shows the behavior of the residual rms values and of the correlation coefficients as a function of the dipole parameter. The different behavior for the $\mathrm{M} i_{S} /+10$ series can be easily seen. For other depths, both the residual rms values and the correlation coefficients converge toward a limit. The deeper the sources are, the sooner this limit is approached. Similarly, for deeper sources, the curves of both residual rms values and correlation coefficients lie closer together. After $i_{s}=21$, it is difficult to distinguish between the $\mathrm{M} i_{s} /-10, \mathrm{M} i_{s} /-20, \mathrm{M} i_{s} /-30$, and $\mathrm{M} i_{s} /-40$ series.

\subsection{M23/-20/14 Model}

[39] The preferred model is M23/-20/14, based on the following arguments. The depth of the magnetized layer $(-20 \mathrm{~km})$ is consistent with both low- and high-altitude based solutions. The dipole parameter is the maximum we
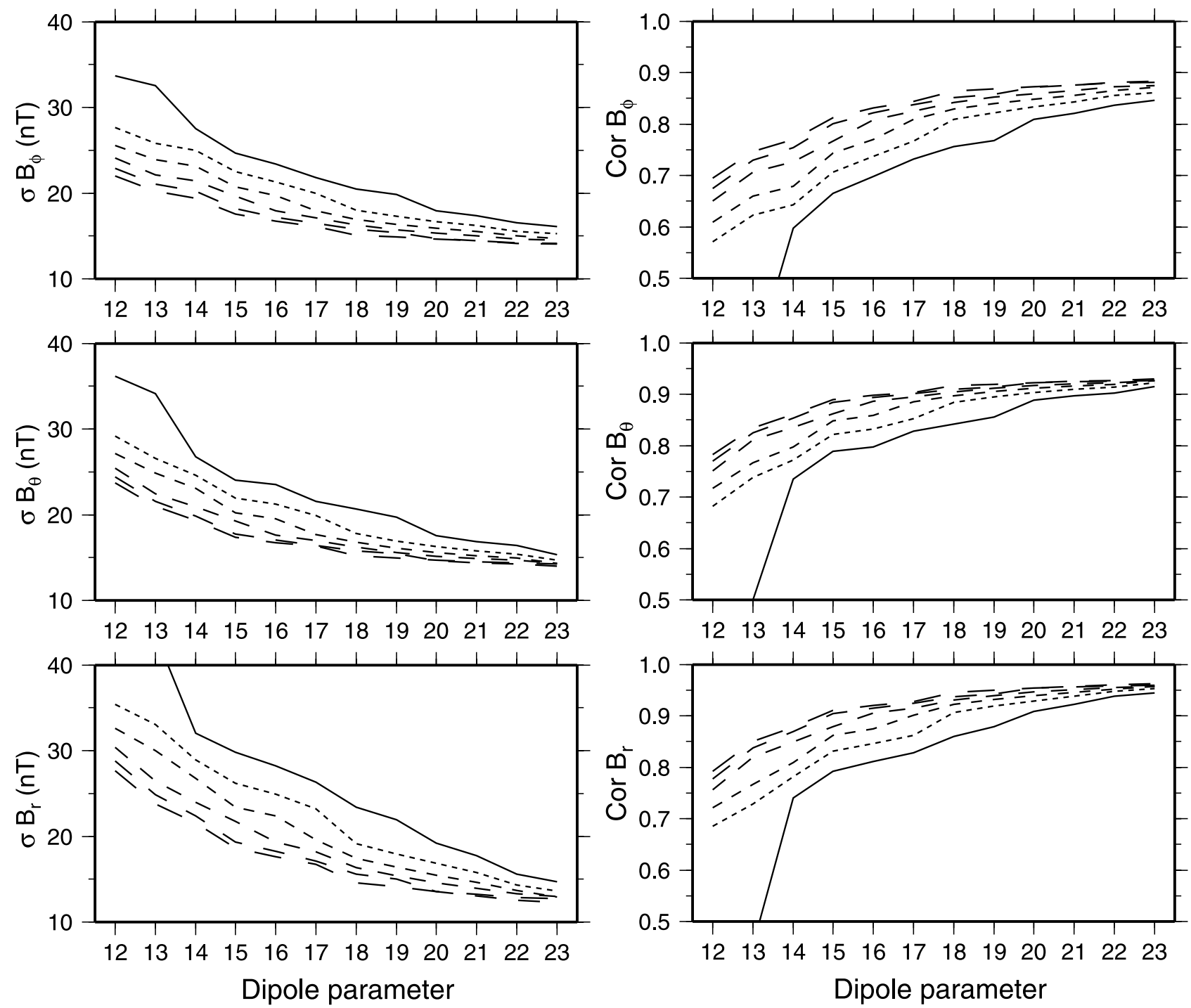

Figure 2. Left Panel: evolution of residual rms values with respect to the dipole parameter. Right panel: evolution of correlation coefficients with respect to the dipole parameter. From solid to long dashed line, depths $=+10,0,-10,-20,-30$ and $-40 \mathrm{~km}$, respectively. From bottom to top, $B_{r}, B_{\theta}, B_{\phi}$. Relative evolution of the residuals is $1.0 \%$. 
Table 2. Error Distribution in the $\pm 1 \sigma$ and in the $\pm 2 \sigma$ Range for the M23/-20/14 Model

\begin{tabular}{cccccccc}
\hline & \multicolumn{3}{c}{$\mathrm{AB}, \mathrm{SPO}$} & & \multicolumn{3}{c}{$\mathrm{MO}$} \\
\cline { 2 - 4 } \cline { 6 - 8 } Error Range, nT & $B_{r}$ & $B_{\theta}$ & $B_{\phi}$ & & $B_{r}$ & $B_{\theta}$ & $B_{\phi}$ \\
\hline 3 & $26.0 \%$ & $17.5 \%$ & $16.7 \%$ & & $66.5 \%$ & $53.1 \%$ & $49.1 \%$ \\
6 & $46.6 \%$ & $32.6 \%$ & $32.1 \%$ & & $92.5 \%$ & $84.7 \%$ & $82.3 \%$ \\
\hline
\end{tabular}

can use for a global problem. However, the evolution of the residual rms values with respect to the dipole parameter showed that $i_{s}=23$ is a reasonable compromise between the quality of the fit to the data, the number of data the model derives from, and the number of dipoles.

[40] The distribution of the residuals of this particular model are given in Table 2. The residuals of all three components are dominantly large scale. Rms values between high-altitude, $\mathrm{MO}$, observations and predictions are 3.3, 4.3 and $4.5 \mathrm{nT}$ for $B_{r}, B_{\theta}$ and $B_{\phi}$, respectively, and 19.2, 20.7 and $21.1 \mathrm{nT}$ for the low-altitude, AB/SPO data sets. All these rms values are comparable to those computed earlier for the low-to-low and high-to-high cases.

[41] The contrast between low-altitude and high-altitude residuals, and between the radial and horizontal components is not surprising. We did not try to remove or model any external magnetic fields. These are likely concentrated in the horizontal components [Krymskii et al., 2002; Vennerstrom et al., 2003]. This is supported by looking at the behavior of the high-altitude residuals with respect to latitude (not shown). The $B_{\theta}$ residuals show an equatorial symmetry between $\pm 60^{\circ}$ latitude, with positive residuals across the equator, and negative residuals at high latitudes. The $B_{\phi}$ residuals show an asymmetric hemispheric behavior, with positive residuals for Northern latitudes and negative residuals for Southern latitudes. No clear trend can be extracted for near-polar latitudes nor for the $B_{r}$ component. Residuals range between $\pm 10 \mathrm{nT}$ near $400-\mathrm{km}$ altitude; at this altitude there is no geographical correlation between the largest residuals and magnetic anomalies.

[42] We computed the parameter error covariance matrix [Purucker et al., 1996, equation (6)] of different dipole solutions, including the one presented in this paper and others similar to the solution of Purucker et al. [2000]. In general, the magnetization matrices exhibit a single dominant eigenvector, carrying $60 \%$ of the variance. Examination of the associated variance reveals regions where small perturbations to the observations would produce correlated, or anti-correlated, changes in nearby groups of dipoles. In the case of the radial magnetization solution of Purucker et al. [2000], and in general where one magnetization component is derived from a single observation component, the largest positive and negative correlations occur in areas where low-altitude observations are missing. This pattern disappears as other observation and magnetization components are added. Significant positive and negative correlations still exist in the dipoles in our preferred model but are located above the areas where the largest magnetization are found, i.e., Terra Cimmeria and Terra Sirenum. The formal error associated with our model is lower than $0.1 \mathrm{~A} / \mathrm{m}$. Maps of the magnetization variance and covariance are given in the electronic supplement ${ }^{1}$.

\footnotetext{
${ }^{1}$ Auxiliary materials are available at $\mathrm{ftp} / / / \mathrm{ftp}$.agu.org/apend/je/ 2003JE002048.
}

[43] Let us now compare our model to previously published ones. The FSU-90 model [Cain et al., 2003] is a spherical harmonic model, up to degree and order 90. It was derived from $\mathrm{AB}, \mathrm{SPO}$ and $\mathrm{MO}$ data sets, only the last one being night-side selected. The authors did not use binned data, but rather decimated data along orbit tracks, in order to get as uniform a geographical distribution as possible. The McGill-50 model [Arkani-Hamed, 2002] is another spherical harmonic model, up to degree and order 50. This model was computed using a two-step approach. First, models were computed using only binned, high-altitude MO measurements. Second, these models were compared to low-altitude data based models [Arkani-Hamed, 2001a]. The final 50-degree and order model was then derived by using a covariance technique [Arkani-Hamed et al., 1994], retaining only the average of the covarying spherical harmonic coefficients.

[44] To compare observations and models we selected some AB orbits between day 341 of 1998 and day 28 of 1999. All retained orbits have periapsis near $100-\mathrm{km}$ altitude, with absolute measured field components larger than 1000 nT. 38 of the 39 orbits contain measurements made above Terra Cimmeria and Terra Sirenum. We predict the field values using M23/-20/14, McGill-50 and FSU-90 models. We then compute the rms differences between observations and predictions as a function of altitude. For a given altitude, the residual rms values are computed using all measurements made above that particular altitude. We present in Figures $3 \mathrm{a}-3 \mathrm{c}$ the statistics for $B_{r}, B_{\theta}$ and $B_{\phi}$, respectively. While both FSU-90 and M23/-20/14 give similar results, with FSU-90 slightly better below 200-km altitude, the McGill-50 model has much higher residual rms values. To test whether the difference comes from the different maximum degree and order of the model compared to the FSU-90, we then plot in Figures $3 \mathrm{~d}-3 \mathrm{f}$ the residuals computed for a truncated (at degree and order 50) version of FSU-90 which we denote FSU-50. The fit of both McGill-50 and FSU-50 is similar, but the FSU-50 still gives slightly lower residuals. We also tried to understand why the residuals computed with our model start to diverge from those computed with FSU-90 below 160- to $180-\mathrm{km}$ altitude. We thus computed two other models, relying only on vertical dipoles (only the first term of the right member of equations (A11), (A12), and (A13) are kept), denoted M23/ $-20 / 08\left(M_{r}\right)$ and $M 40 /-20 / 17\left(M_{r}\right)$. In each case, the iteration number was selected using the technique described previously. Their residual rms values are also shown in Figures $3 \mathrm{~d}-3 \mathrm{f}$. Two conclusions can be drawn from these tests. First, the M23/-20/08( $\left.M_{r}\right)$ model gives similar rms to those of the FSU-50 and McGill-50 models. Residual rms values start to increase drastically for altitudes lower than $200 \mathrm{~km}$. Second, the residuals computed using the M40/-20/ $17\left(M_{r}\right)$ model are almost identical to those of the FSU-90 model, with divergence starting below $120-\mathrm{km}$ altitude.

[45] M23/-20/14 model has a mean horizontal spacing of $173 \mathrm{~km}$, comparable to the $160-180-\mathrm{km}$ altitude range where the residuals computed using model M23/-20/14 start to increase. Following Mayhew [1979], it is difficult to predict data below an altitude equal to the mean horizontal resolution. M23/-20/08( $\left.M_{r}\right)$ and M40/-20/17( $\left.M_{r}\right)$ models have mean horizontal spacings of 173 and $97 \mathrm{~km}$, respectively. The divergence is observed near 200 and $120 \mathrm{~km}$, respectively. 


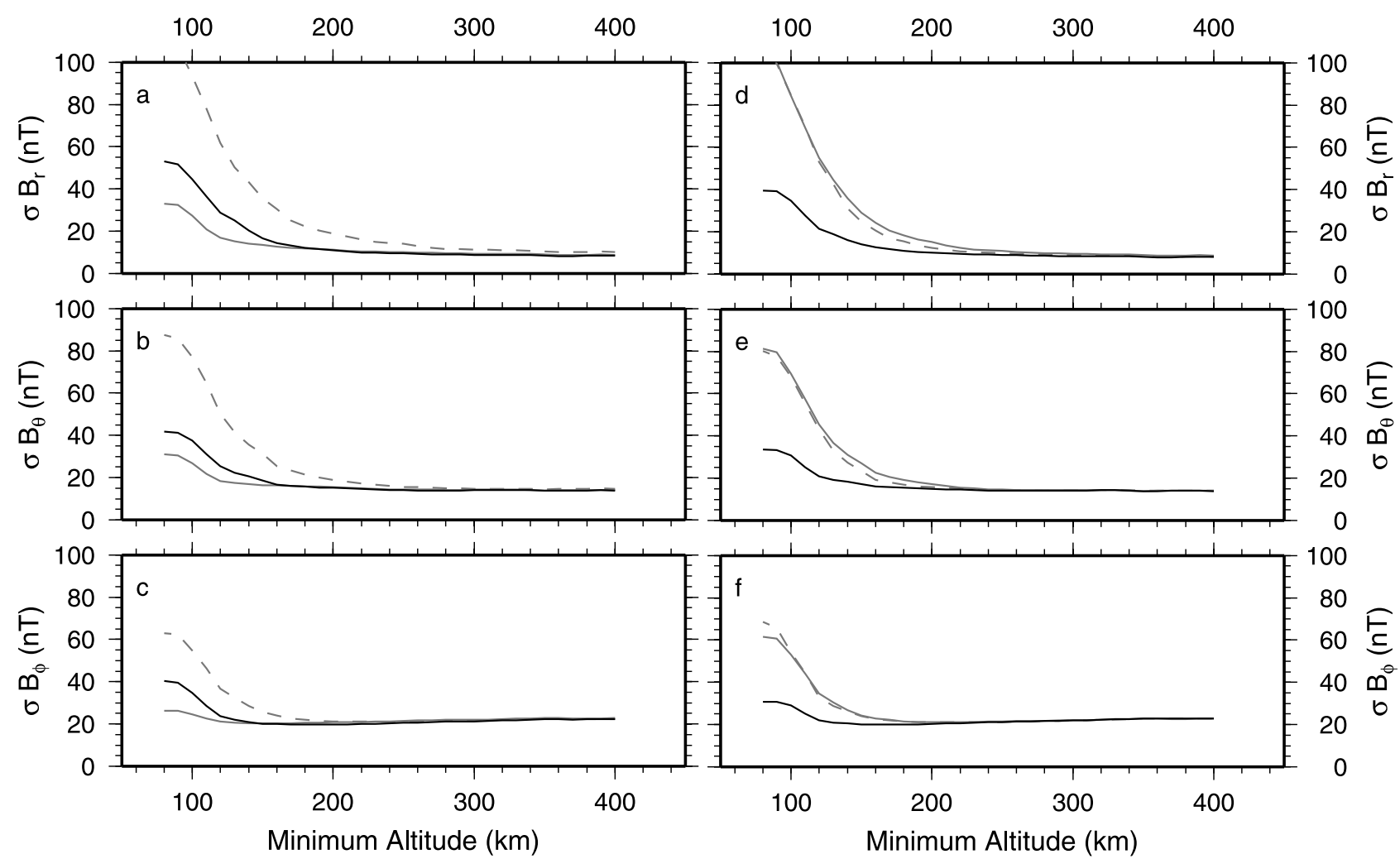

Figure 3. Residual rms values between selected orbits and prediction from model. From a to c, using M23/-20/14 (black solid line), McGill-50 (gray dashed line), and FSU-90 (gray solid line). From d to f, using M40/-20/17( $\left.M_{r}\right)$ (black solid line), M23/-20/08( $\left.M_{r}\right)$ (gray dashed line), and FSU-50 (gray solid line).

This is a little higher than what one might expect, but the pure radial dipoles certainly introduced a bias in the solution. Our model appears to be less accurate for the shortest wavelengths at low altitude than spherical harmonic models. But it also suggests a distribution of the magnetic sources.

\section{Discussion}

[46] We now will focus on a global view of the Martian magnetic field, and emphasize correlations between impact craters and the magnetization model.

\subsection{Magnetic Field}

[47] We plot in Figure 4 the three components of the magnetic field at $200 \mathrm{~km}$ altitude predicted by the M23/ $-20 / 14$ model. The magnetic field is weak (within $\pm 10 \mathrm{nT}$ ) almost everywhere North of the crustal dichotomy (also plotted in Figure 4). Only two features North of the crustal dichotomy are to be noted, located near $\left(70^{\circ} \mathrm{N}, 30^{\circ} \mathrm{E}\right)$ and $\left(45^{\circ} \mathrm{N}, 185^{\circ} \mathrm{E}\right)$. The largest magnetic anomalies are observed over Terra Cimmeria and Terra Sirenum. The largest craters, Isidis Planitia, Argyre and Hellas, are not associated with large magnetic anomalies, nor are the largest volcanoes of the Northern hemisphere (Tharsis Montes, Olympus Mons, and Elysium Mons). At least two end-member scenarios can be considered to explain this lack of anomalies. In the first scenario, both the giant impacts and the giant volcanic features took place after the Martian dynamo turned off. The lithosphere would have been demagnetized, because of the thermal and shock effects. In the second scenario, the impacts and the volcanic edifices took place before the Martian dynamo turned on. But this later scenario is less likely, as both the giant impacts and the major volcanic edifices are probably younger than the terranes of the Terra Cimmeria and Terra Sirenum areas, where we see the largest magnetic anomalies.

[48] We computed the magnetic field at $200-\mathrm{km}$ altitude on a $0.5^{\circ}$ grid. The extrema (rounded to the nearest tens) of the predicted magnetic field are $-410 /+610 \mathrm{nT}$ for $B_{r}$, $-540 /+460 \mathrm{nT}$ for $B_{\phi}$ and $-300 /+270 \mathrm{nT}$ for $B_{\phi}$. These values are very similar to those predicted by the FSU-90 model: $-410 /+640 \mathrm{nT}$ for $B_{r},-570 /+450 \mathrm{nT}$ for $B_{\theta}$ and $-290 /+270 \mathrm{nT}$ for $B_{\phi}$. We downward continued our predicted magnetic field to lower altitudes. Because the input dipole mesh has a low resolution, such predictions will have significant uncertainties. However, such numbers may give an idea of the fields which might be expected in follow-on missions, if account is taken of the missing shorter-wavelength features. At 100-km altitude, the radial magnetic field encompasses the range $\pm 2200 \mathrm{nT}$, while $B_{\theta}$ and $B_{\phi}$ range between $\pm 1600 \mathrm{nT}$ and $\pm 1000 \mathrm{nT}$, respectively. We compared these values to those predicted by other models. The FSU-90 model predicts amplitudes at $100 \mathrm{~km}$ altitude of $2300 \mathrm{nT}, 2000 \mathrm{nT}$ and $1200 \mathrm{nT}$ for $B_{r}, B_{\theta}$ and $B_{\phi}$, respectively. We also calculated the surface fields predicted from our model, after first converting the equivalent source representation to a spherical harmonic one (it is impossible to predict directly from the equivalent sources solution 
a

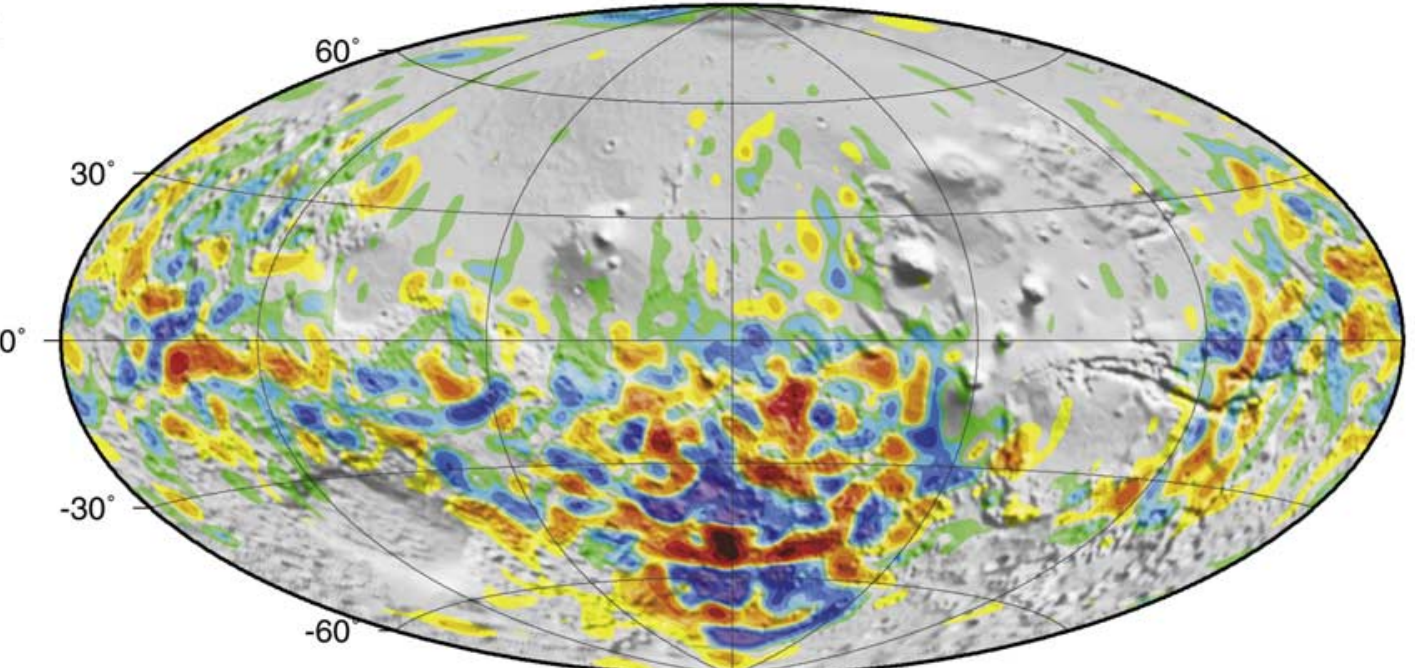

$\mathrm{B}_{\mathrm{r}}(\mathrm{nT})$

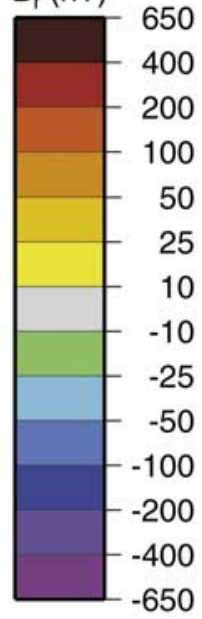

b

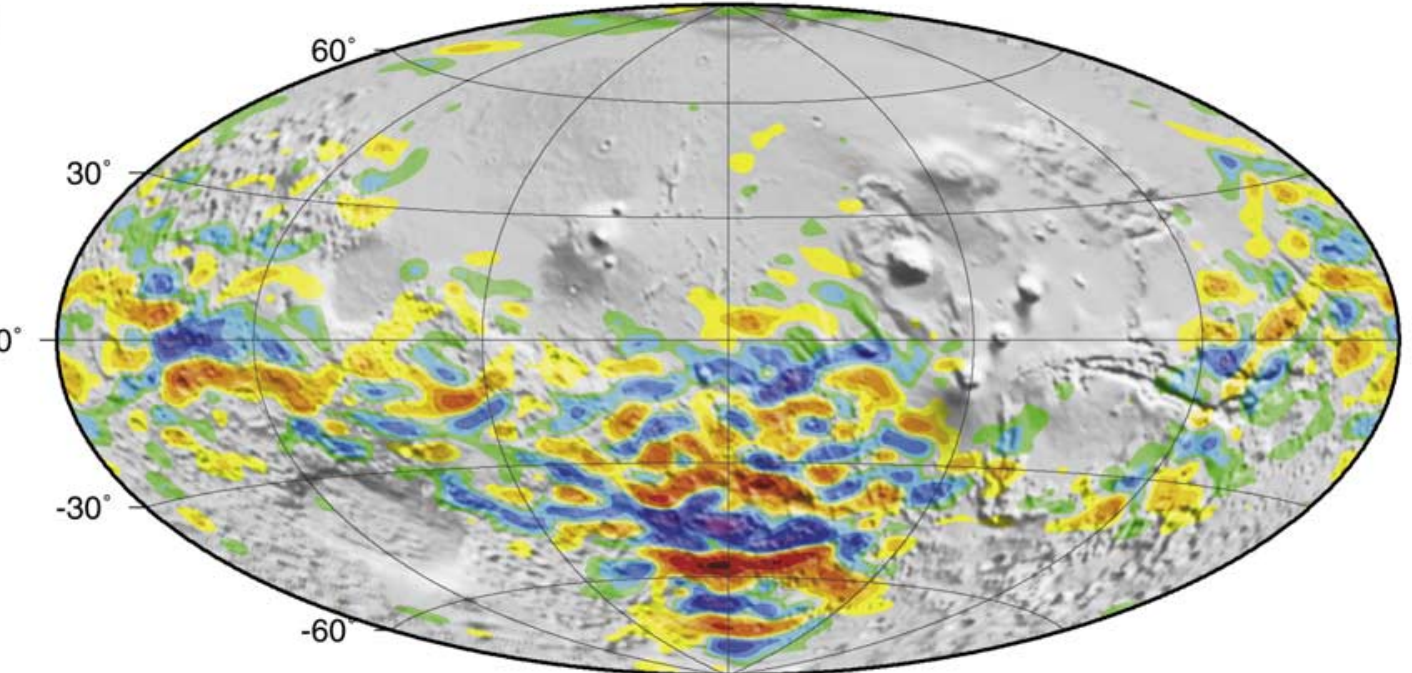

$\mathrm{B}_{\theta}(\mathrm{nT})$

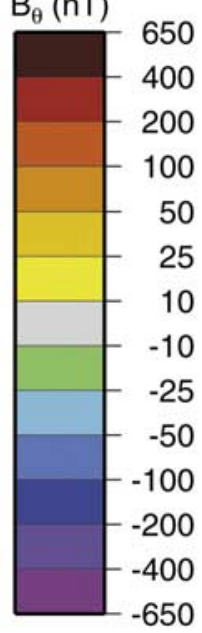

C

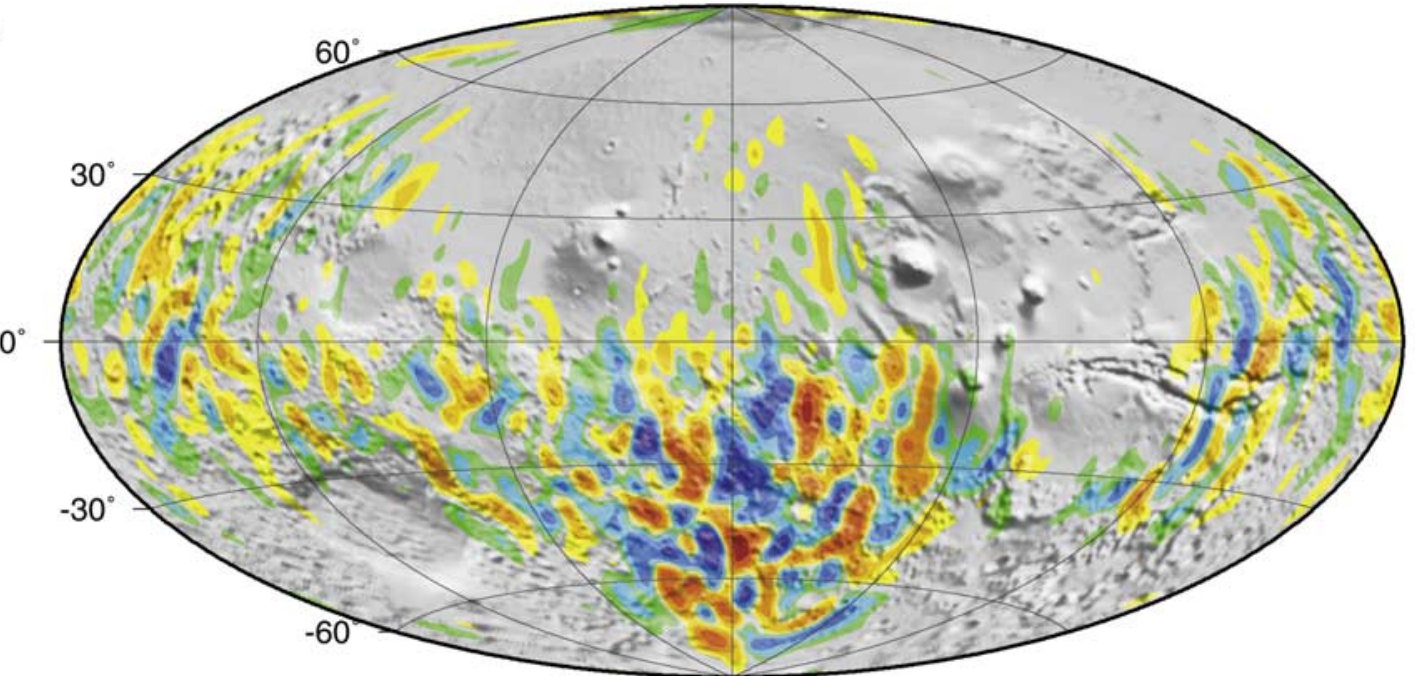

$\mathrm{B}_{\phi}(\mathrm{nT})$

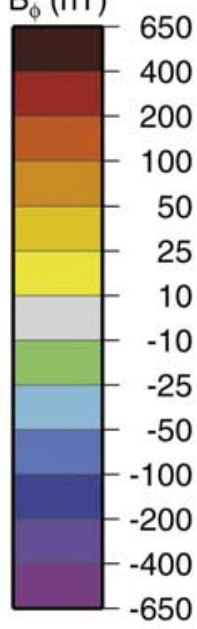

Figure 4. Predicted magnetic field at 200-km altitude, from M23/-20/14.

magnetic measurements below an altitude equal to the mean horizontal spacing of our model). The predicted scalar magnetic field ranges up to $6000 \mathrm{nT}$. The components predicted by the McGill-50 model range between \pm 2600 nT [see Arkani-Hamed, 2001a, Plate 1], much lower than our results. This $6000 \mathrm{nT}$ range sets lower limits on the surface Martian magnetic field, because our model does not take into account magnetic fields with wavelength content 


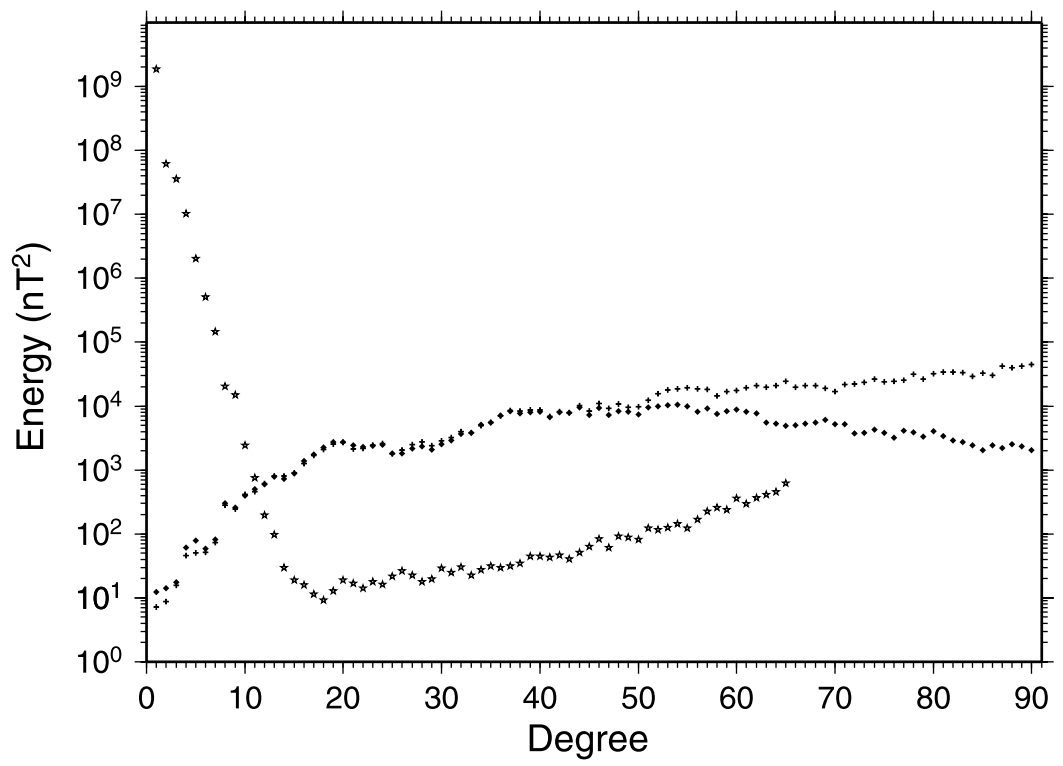

Figure 5. Energy spectra of the Earth's magnetic field from Sabaka et al. [2002] model (stars), and of Mars' magnetic field from our model (black diamonds) and from Cain et al. [2003] model (crosses).

below $170 \mathrm{~km}$. FSU-90 model gives a $12000 \mathrm{nT}$ range. It is likely that on Mars' surface, in the Terra Sirenum and Terra Cimmeria regions, the amplitude of the magnetic field is very similar to the Earth's magnetic field $( \pm 50000 \mathrm{nT})$. However, the geomagnetic field is mostly of core origin, and the Earth's lithospheric field is commonly in the $\sim \pm 15 \mathrm{nT}$ at $400 \mathrm{~km}$ altitude [Maus et al., 2002].

[49] We present in Figure 5 a comparison of the energy spectra of the Earth's and Mars' magnetic fields, using CM3 model [Sabaka et al., 2002] for the Earth, and FSU-90 [Cain et al., 2003] and our converted model for Mars. Between degrees 15 and 50, there is a difference of $10^{2}$ between the spectra of Earth and Mars. The two Mars' models are consistent up to degrees 50/55. Thereafter our model is less energetic, perhaps due to the truncation that our equivalent dipole model carries. Indeed, our dipole mesh has a mean resolution of $2.92^{\circ}$, i.e., $173 \mathrm{~km}$. The spherical harmonic model wavelength [Ravat et al., 2002] can be expressed as

$$
\lambda=\left[\frac{8 \cdot \pi \cdot R^{2}}{n \cdot(n+1)}\right]^{1 / 2}
$$

where $n$ is the maximum degree and $R$ the mean radius of Mars, here $3393.5 \mathrm{~km}$. The maximum degree corresponding to twice the input dipole mesh resolution is 49 . Above this degree, the equivalent source fields become laterally correlated [Voorhies et al., 2002], which could explain the decrease of the magnetic spectrum. Thus any conclusion drawn from the spectrum of our model for $n$ higher than 50 would be hazardous.

\subsection{A Magnetization Map}

[50] Given our assumptions (40-km thick iso-volume blocks of homogeneously magnetized material), the magnetization ranges $-9.0 /+11.5,-7.8 /+11.3$ and $-6.2 /+6.7 \mathrm{~A} / \mathrm{m}$ for $M_{r}, M_{\theta}$ and $M_{\phi}$, respectively. Of course these values are not absolute, because of the non uniqueness of the problem, but they are representative of the expected magnetization contrasts in the Martian lithosphere. Interestingly enough, these figures are very consistent with the ones derived by Parker [2003]. In his study, he computed what would be the minimum magnetization responsible for some of the largest magnetic anomalies on Mars. Assuming a 50-km thick magnetized layer, the intensity of the magnetization would be at least $4.76 \mathrm{~A} / \mathrm{m}$.

[51] The radial magnetization values are lower than those computed by Purucker et al. [2000], but their model relied only on the low-altitude, geographically sparse, data set. They restrained their model to purely radial magnetization, did not try to model the horizontal magnetic components and used a preliminary binned version of the $A B$ data set. Furthermore, they considered a $1.9^{\circ}$ mean spacing for the dipoles, which made their magnetized bodies smaller than ours (thus leading to larger magnetizations).

[52] We plot in Figure 6 the three components of the magnetization from the M23/-20/14 model. The anomaly and magnetization map are in good accordance. High magnetization values are mostly located South of the crustal dichotomy, with the exception of the negative $M_{\phi}$ circular feature, near $70^{\circ}$ North latitude. This is likely the signature of external magnetic fields, or of their induced counterpart.

[53] We superimposed on the magnetization maps (Figure 6) the locations of the circular crater rims with diameter larger than $300 \mathrm{~km}$. A list of the craters, together with their location and radius is given in Table 3 . It is likely that impacts demagnetized the Martian lithosphere, as it has been observed on the Moon [Halekas et al., 2002, and references therein]. The ratio between the crater radius and the excavation depth is quite well defined, but this depends on the complexity of the crater, or on the geological layout. For instance, Garvin et al. [2000] give a mean depth-todiameter ratio of $0.053 \pm 0.04$ for non-polar, relatively small impact features. Of course, this mean ratio cannot be extended to the largest craters. Hellas, a 2000-km-diameter crater, is only $9 \mathrm{~km}$ deep. Also, this ratio is not the one for the demagnetization depth. It is likely that the destructive effects 
a

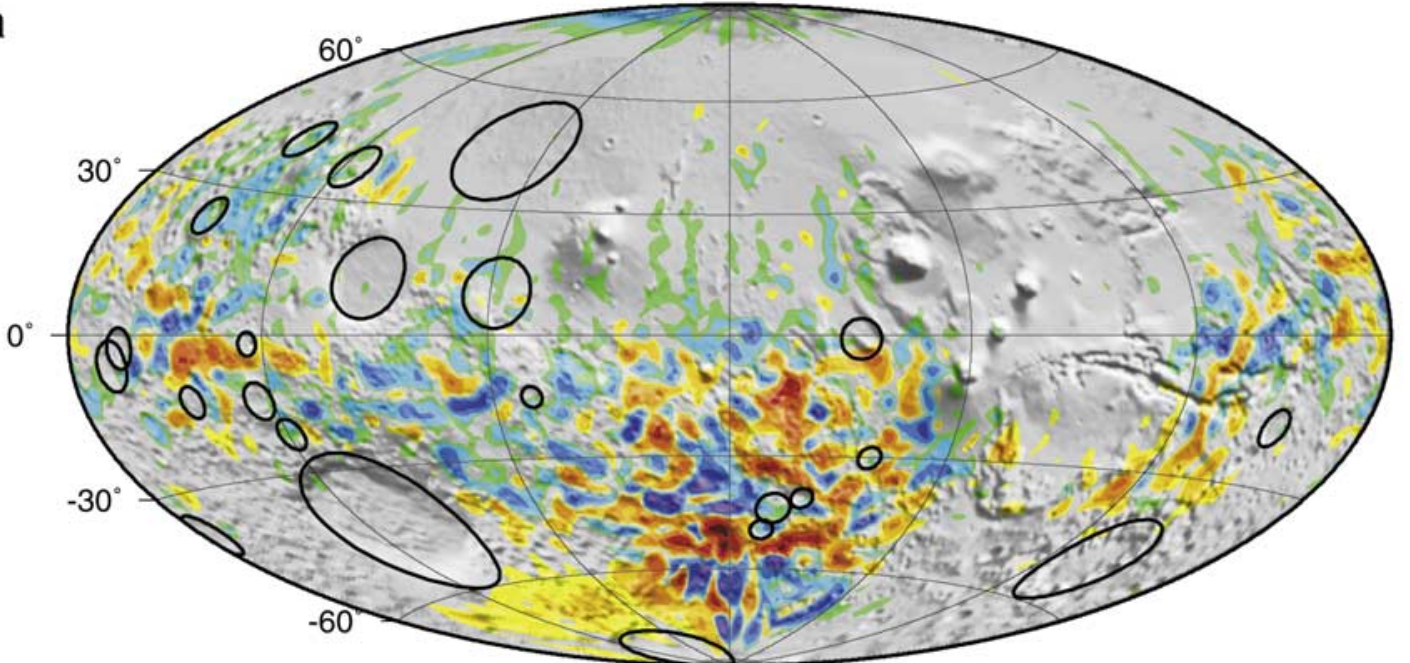

b

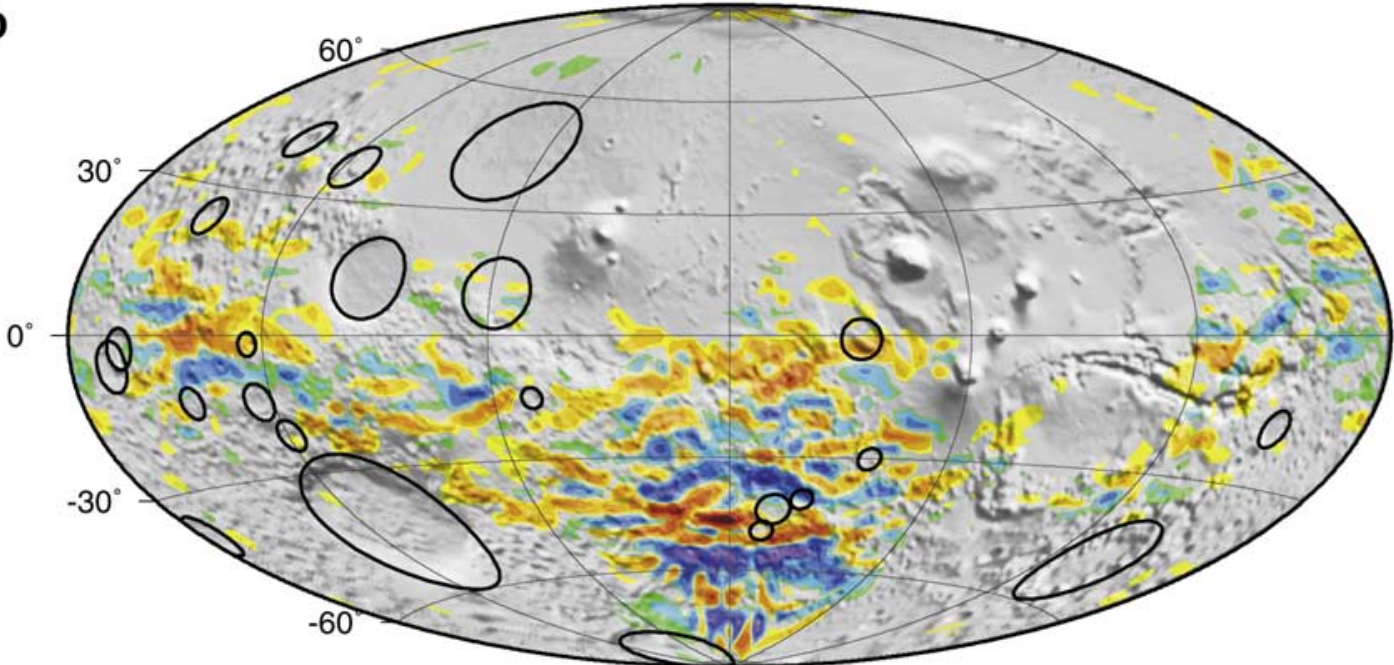

C

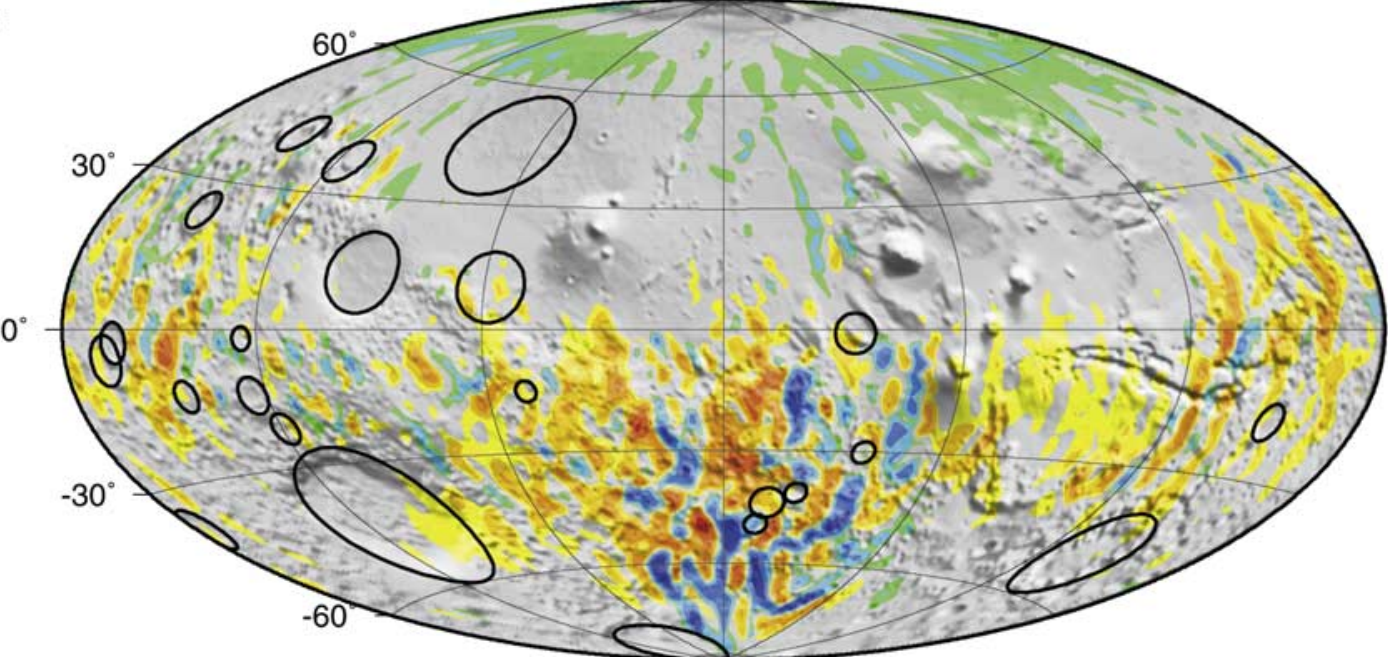

$M_{r}(A / m)$

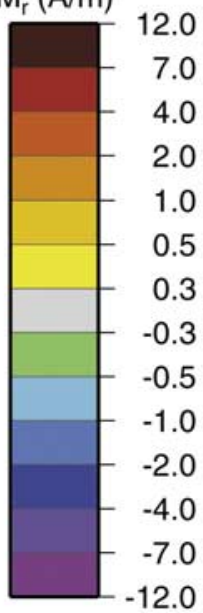

$M_{\theta}(A / m)$

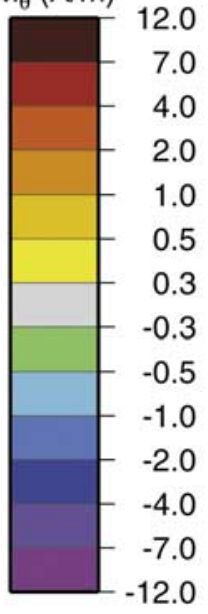

$M_{\phi}(A / m)$

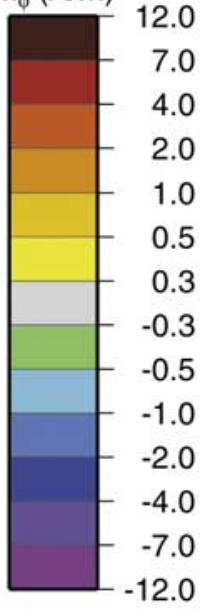

Figure 6. Magnetization from M23/-20/14. These maps were obtained using Delaunay triangulation of GMT [Wessel and Smith, 1991].

of the impact extend below the crater depth. Such phenomena were observed on the Earth [Pilkington and Grieve, 1992], although some terrestrial craters are also characterized by shock and/or thermal remanence. In their study,
Nimmo and Gilmore [2001] assumed a 0.06 demagnetization depth-to-diameter ratio. This value is similar to but larger than the one given by Garvin et al. [2000]: the demagnetization depth is at least equivalent to the excavation depth. 
Table 3. Crater Names and Characteristics ${ }^{\mathrm{a}}$

\begin{tabular}{lrrc}
\hline \multicolumn{1}{c}{ Crater Name } & Lat., ${ }^{\circ}$ & Lon., ${ }^{\circ}$ & Radius, km \\
\hline Hellas & -43.0 & 69.0 & 1000 \\
Utopia & 45.0 & 110.0 & 750 \\
Argyre & -49.5 & 318.0 & 600 \\
Isidis & 13.0 & 87.0 & 550 \\
South of Hephaestus & 10.1 & 121.6 & 500 \\
Mangala & -0.9 & 212.5 & 295 \\
Overlapping Schiaparelli & -5.8 & 13.6 & 280 \\
South of Renaudot & 37.5 & 63.5 & 280 \\
West of LeVerrier & -37.9 & 2.6 & 250 \\
South of Lyot & 41.6 & 38.0 & 240 \\
Huygens & -14.0 & 55.8 & 235 \\
Sirenum & -43.5 & 193.6 & 230 \\
Schiaparelli & -2.5 & 16.7 & 230 \\
Ladon & -18.4 & 330.6 & 220 \\
Cassini & 24.0 & 31.8 & 220 \\
Antoniadi & -21.5 & 60.8 & 200 \\
Tikhonravov & -13.5 & 35.8 & 190 \\
Kovalsky & -30.2 & 218.5 & 160 \\
Copernicus & -49.2 & 190.8 & 150 \\
Herschel & -14.9 & 129.9 & 150 \\
Newton & -40.8 & 201.9 & 150 \\
Schroeter & -1.9 & 55.6 & 150 \\
\hline
\end{tabular}

${ }^{a}$ Lat. and Lon. are latitude and East longitude. Radius is rounded to the nearest $5 \mathrm{~km}$.

Nimmo and Gilmore [2001] observed an apparent weaker magnetic field associated with craters larger than $500 \mathrm{~km}$ in diameter, and inferred a thickness of $35 \mathrm{~km}$ for the demagnetized layer (or for the thickness of the magnetic crust).

[54] Our magnetization model seems to suggest some correlation between weak magnetized regions and $300-\mathrm{km}$ diameter and larger craters. Of course, we did not expect to see exact, circular signatures of the crater rims in the magnetization map, because of the size of our initial dipole mesh. The largest craters, Hellas, Argyre, Utopia and Isidis, do not show any strong magnetization. Using the MGS Electron Reflectometer measurements, Mitchell et al. [2002] showed that the magnetic field above the Hellas basin was weak, but non zero. Our model does not seem to support this hypothesis (except for the $M_{\phi}$ component on the Eastern part of the basin), but we cannot reject it. Above only one large impact crater (South of Hephaestus, 1000-km diameter), there are some small magnetized features, especially near its Southern rim. However, the scale of these anomalies is very small compared to the size of the crater. Using preliminary AB data, Nimmo and Gilmore [2001] pointed out that no radial magnetic features could be associated with craters larger that $500-\mathrm{km}$ diameter. On the magnetization map, there is a clear association between non-magnetized and impact areas, especially over some of these craters, like Schroeter (especially for $M_{r}$ on its South rim), Newton $\left(M_{r}\right.$ and $\left.M_{\phi}\right)$, Copernicus $\left(M_{r}\right.$ and $\left.M_{\theta}\right)$, Herschel, Kovalsky and Tikhonravov $\left(M_{r}, M_{\theta}\right.$ and $\left.M_{\phi}\right)$. In order to further estimate these correlations, or to give actual metrics on these correlations, one would need to consider a finer dipole mesh (down to $1.9^{\circ}$ or so).

[55] The above correlations have implications for either the thickness of the magnetized layer or the demagnetization depth. It could also help to better understand the shock demagnetization process [Halekas and Lin, 2003]. It seems that large impact craters (300-km diameter and larger) are associated with locally weaker magnetized crust. If we consider a magnetic thickness of 35 to $50 \mathrm{~km}$, this would imply a depth-to-diameter ratio between 0.11 and 0.17 , which is twice the mean value of 0.06 adopted by Nimmo and Gilmore [2001]. Their value would imply a magnetized thickness of $18 \mathrm{~km}$, leading to more than doubling of the magnetization range to $\pm 26.6 \mathrm{~A} / \mathrm{m}$.

\subsection{A Quest for Magnetization Directions and Paleopoles}

[56] A knowledge of magnetization directions and paleopoles would provide critical information on the tectonic evolution for both local and global studies of the Martian crust. The inference of paleopoles from magnetization directions relies on the assumption that the magnetic field recorded by the rocks was dominantly dipolar. Inferences on possible polar wandering on Mars from this information also relies on the approximate coincidence of the ancient magnetic and spin poles.

[57] Determination of magnetization directions on the Earth, and by extension, on Mars, relies on the following techniques, listed in order of decreasing reliability: 1) in-situ determination from the rocks themselves, 2) inversions of magnetic field observations over a body of known geometry, 3) inversions of magnetic field observations over isolated dipolar sources, and 4) inversions of magnetic field observations over multiple, and overlapping, sources.

[58] Technique \#1 yields unequivocal information about magnetization direction, and may also yield information on other, superimposed magnetizations which have different coercivity spectra. This can be applied to Martian meteorite samples, taking into account that they are unoriented and have been subject to additional thermal and shock events on their way to the Earth. Technique \#2 yields generally reliable results if the magnetization in the known source body can be assumed homogeneous. Because the Martian magnetic layer is generally deep, the application of this method to Mars is extremely limited. Technique \#3 is applied by first searching for the optimum location of the isolated magnetized body [Arkani-Hamed and Boutin, 2003; Richmond and Hood, 2003], then solving for its directions. If the search is successful in locating the dipole, and if the source body is homogeneous, the technique can yield reliable results. The application of this technique is again very limited, with only a dozen or so examples. Technique \#4, of which the results in this paper are an example, can be applied anywhere, including the most intensely magnetized terranes in the Southern hemisphere where the other techniques are impossible to apply for now. The paleopole locations derived using this technique should be considered as possible solutions, and in light of results from the other techniques. We computed the location of the paleopoles associated with dipoles whose total magnetization exceeds $4 \mathrm{~A} / \mathrm{m}$. This represents 116 paleopoles out of a total of 4840 dipoles. As expected, these dipoles are mostly located over Terra Cimmeria and Terra Sirenum, although some are also located in Terra Meridiani. A few observations can be made. First, the poles do not show a dipolar distribution or clustering, in contrast to the one pointed out by Arkani-Hamed and Boutin [2003]. Second, immediately adjacent dipoles frequently have very different paleopoles. This latter characteristic is also present in other studies (see poles associated to anomalies 11 and 16 in Figure 2 of Arkani-Hamed and Boutin [2003]). 
Table 4. Inclination $I$ and Declination $D$ at the Location of the 10 Anomalies Described by Arkani-Hamed [2001b] ${ }^{\mathrm{a}}$

\begin{tabular}{|c|c|c|c|c|c|c|}
\hline \multirow[b]{2}{*}{ Anomaly } & \multirow[b]{2}{*}{ Lon. } & \multirow[b]{2}{*}{ Lat. } & \multicolumn{2}{|c|}{$\begin{array}{c}\text { Arkani-Hamed } \\
{[2001 \mathrm{~b}]} \\
\end{array}$} & \multicolumn{2}{|c|}{ This Study } \\
\hline & & & $I$ & $D$ & $I$ & $D$ \\
\hline M1 & 20 & -4 & 69 & -20 & 82 & -172 \\
\hline M2 & 31 & 15 & 76 & -116 & 79 & 138 \\
\hline M3 & 27 & 65 & 29 & -92 & 22 & -11 \\
\hline M4 & 66 & -5 & -75 & 149 & -80 & -26 \\
\hline M5 & 69 & -15 & 59 & 29 & 88 & 45 \\
\hline M6 & 103 & -27 & 80 & -6 & 73 & -54 \\
\hline M7 & 214 & -5 & -71 & -180 & -67 & -172 \\
\hline M8 & 309 & -25 & -78 & 90 & -71 & -109 \\
\hline M9 & 322 & -1 & 76 & 88 & 80 & 122 \\
\hline M10 & 344 & 2 & -68 & 114 & -86 & -36 \\
\hline
\end{tabular}

${ }^{\mathrm{a}}$ All values in ${ }^{\circ}$.

[59] Our model, however, compares well to other models. Whaler and Purucker [2003], in a preliminary study, used only radial field data to define a continuous planet-wide magnetization function. They find that six of the ten paleomagnetic poles of Arkani-Hamed and Boutin [2003] are within $30^{\circ}$ of their paleomagnetic poles. Table 4 shows that the magnetization inclinations calculated from Technique \#3 [Arkani-Hamed, 2001b] are within $10^{\circ}$ of the inclinations calculated in this paper, in seven of the ten cases, and are always within $30^{\circ}$. The declinations are more dispersed, as would be expected for a situation in which nine of the ten isolated dipoles have steep inclinations. The values we computed are stable, as pointed out by some tests we performed (see Appendix B). The correspondence between techniques \#3 and \#4, and between the three studies, gives additional confidence in the magnetization direction determination from the isolated dipoles. The magnetization direction solutions in areas of overlapping magnetic sources are probably more complicated than shown in our results, but will be useful in guiding tectonic or geologic interpretations [Whaler and Purucker, 2003].

\section{Conclusions}

[60] Because of the non-uniqueness of the problem, it is impossible to infer the absolute magnetization of the Martian lithosphere. This is why we tested numerous input dipole mesh parameters and depths. The results and interpretations given in that study are based on the finest input dipole mesh we were able to use, a mean spacing of $2.92^{\circ}$. The numerous tests we performed, using different mesh resolution and dipole depth, indicate that the results are reliable in terms of magnetization contrasts. A good agreement with previously published models is observed.

[61] The Martian magnetic field is undoubtedly of lithospheric origin. Its amplitude at $200-\mathrm{km}$ altitude is $\pm 650 \mathrm{nT}$. At the surface, it could be up to a few tens of thousands of nT. The magnetization we computed that would produce such a magnetic field ranges between $\pm 12 \mathrm{~A} / \mathrm{m}$ for a $40-\mathrm{km}$ thick magnetic crust. The correlations observed between the magnetization contrasts and the impact craters indicate that this thickness could be as low as $18 \mathrm{~km}$. In this case, the magnetizations would range between $\pm 25 \mathrm{~A} / \mathrm{m}$. In order to produce such magnetizations, there would need to be a high content of magnetic minerals, magnetized in a coherent and large-scale fashion, in the Martian lithosphere.
[62] We considered sources located $20 \mathrm{~km}$ below the reference radius of Mars of $3393.5 \mathrm{~km}$. We checked that the general behavior observed in our model was present in the M23/+00 and M23/-10 series. Given the Martian ellipticity, the real distance between the surface and the top of our equivalent source layer would range between 5 and $10 \mathrm{~km}$ between $\pm 30^{\circ}$ latitude. Given this, and the possible demagnetization depth, the magnetic crust could be as thin as $10 \mathrm{~km}$, leading to even larger magnetization values.

[63] This magnetization is one order of magnitude larger than that found on the Earth. In a recent study by Rochette et al. [2001], several SNC meteorites were analyzed. Possible candidates to carry the magnetization are pyrrhotite, titanomagnetite or hematite. Hematite was detected at the surface of Mars [Christensen et al., 2001]. It has been suggested that this mineral could carry the martian magnetization [Dunlop and Kletetschka, 2001]. But recent studies pointed out the superficial characteristics and water-related origin of the hematite deposits [Hynek et al., 2002]. Pyrrhotite and titanomagnetite have different Curie points $\left(320^{\circ} \mathrm{C}\right.$ vs. $150-580^{\circ} \mathrm{C}$, depending on the Ti proportion). The Curie isotherm for pure magnetite was $50 \mathrm{~km}$ deep 4 Gyr B.P. [Nimmo and Gilmore, 2001, Figure 4]. That for pyrrhotite was at $25 \mathrm{~km}$. This would be consistent with our deductions for the estimated thickness of the magnetization layer. However, considering the relatively low blocking pressure of pyrrhotite (between 1.6 GPa and 4.5 GPa [Vaughan and Tossell, 1973; Kobayashi et al., 1997; Rochette et al., 2003]), the demagnetization depth to crater diameter ratio could be larger. Although we did not discuss this in our study, the impact-related demagnetization may well extend 3-4 basin radii [Hood et al., 2003]. One might thus expect demagnetized signatures over smaller craters $(\leq 300-\mathrm{km}$ diameter $)$, but the resolution of our model does not allow such correlations to be seen.

[64] Our model is the first global model of the Martian magnetic field that can explain observations in terms of possible magnetization distributions. Our model can thus be used as a tool to predict the magnetic field and to study the magnetic properties of the lithosphere. The limitations are linked to the method we used: (i) we considered a discrete magnetization distribution, with a mean horizontal resolution of $173 \mathrm{~km}$; (ii) the inverse problem is highly non-unique, and our solution is only one possibility of what could be the real situation on Mars. There is, however, a good agreement observed between constant altitude magnetic components predicted by our preferred model and by spherical harmonic analysis. The fit to the data (about $10 \mathrm{nT}$ ), and the mean spacing of our model (about $170 \mathrm{~km}$ ), are both figures that could decrease. Efforts are needed to refine the data selection, increase the model resolution, but also better remove and/or model the external contributions. There is also a crucial need for new low-altitude magnetic measurements, to confirm and extend the present results.

\section{Appendix A: Modeling Scheme}

[65] One can write the potential at $(r, \theta, \phi)$ due to a dipole located at $\left(r_{d}, \theta_{d}, \phi_{d}\right)$ as:

$$
V=\frac{M_{r}\left(r A_{1}-r_{d}\right)-M_{\theta} r B_{1}+M_{\phi} r C_{1}}{l^{3}}
$$


where $l$ is defined in equation (2), and the coefficients are

$$
\begin{gathered}
A_{1}=\cos (\theta) \cos \left(\theta_{d}\right)+\sin (\theta) \sin \left(\theta_{d}\right) \cos \left(\phi-\phi_{d}\right) \\
B_{1}=\cos (\theta) \sin \left(\theta_{d}\right)-\sin (\theta) \cos \left(\theta_{d}\right) \cos \left(\phi-\phi_{d}\right) \\
C_{1}=\sin (\theta) \sin \left(\phi-\phi_{d}\right)
\end{gathered}
$$

[66] Following equation 4, we calculate the partial derivatives of $A_{1}, B_{1}$ and $C_{1}$ [Mayhew et al., 1984; Dyment and Arkani-Hamed, 1998]:

$$
\begin{gathered}
A_{2}=\frac{\partial A_{1}}{\partial \theta}=-\sin (\theta) \cos \left(\theta_{d}\right)+\cos (\theta) \sin \left(\theta_{d}\right) \cos \left(\phi-\phi_{d}\right) \\
B_{2}=\frac{\partial B_{1}}{\partial \theta}=-\sin (\theta) \sin \left(\theta_{d}\right)-\cos (\theta) \cos \left(\theta_{d}\right) \cos \left(\phi-\phi_{d}\right) \\
C_{2}=\frac{\partial C_{1}}{\partial \theta}=\cos (\theta) \sin \left(\phi-\phi_{d}\right) \\
A_{3}=\frac{\partial A_{1}}{\sin (\theta) \partial \phi}=-\sin \left(\theta_{d}\right) \sin \left(\phi-\phi_{d}\right) \\
B_{3}=\frac{\partial B_{1}}{\sin (\theta) \partial \phi}=\cos \left(\theta_{d}\right) \sin \left(\phi-\phi_{d}\right) \\
C_{3}=\frac{\partial C_{1}}{\sin (\theta) \partial \phi}=\cos \left(\phi-\phi_{d}\right)
\end{gathered}
$$

[67] Finally, using the substitutions $D_{1}=r-r_{d} A_{1}, D_{2}=$ $-r_{d} A_{2}, D_{3}=-r_{d} A_{3}, F_{1}=r A_{1}-r_{d}, F_{2}=-r B_{1}$ and $F_{3}=r C_{1}$, we can write the full expression for the magnetic field components:

$$
B_{r}=M_{r} \frac{\frac{3 D_{1} F_{1}}{l^{2}}-A_{1}}{l^{3}}+M_{\theta} \frac{\frac{3 D_{1} F_{2}}{l^{2}}+B_{1}}{l^{3}}+M_{\phi} \frac{\frac{3 D_{1} F_{3}}{l^{2}}-C_{1}}{l^{3}}
$$

$$
B_{\theta}=M_{r} \frac{\frac{3 D_{2} F_{1}}{l^{2}}-A_{2}}{l^{3}}+M_{\theta} \frac{\frac{3 D_{2} F_{2}}{l^{2}}+B_{2}}{l^{3}}+M_{\phi} \frac{\frac{3 D_{2} F_{3}}{l^{2}}-C_{2}}{l^{3}}
$$

$$
B_{\phi}=M_{r} \frac{\frac{3 D_{3} F_{1}}{l^{2}}-A_{3}}{l^{3}}+M_{\theta} \frac{\frac{3 D_{3} F_{2}}{l^{2}}+B_{3}}{l^{3}}+M_{\phi} \frac{\frac{3 D_{3} F_{3}}{l^{2}}-C_{3}}{l^{3}}
$$

[68] The inverse problem can be written as [Purucker et al., 1996, 2000]

$$
\tilde{b}=\tilde{D} x+\tilde{v}
$$

where $\tilde{b}$ is the vector containing the $n$ magnetic observations (or the $3 \times n$ observed magnetic components), $x$ is the vector containing the parameters of the $m$ dipoles (the $3 \times m$ unknowns), and $\tilde{\nu}$ is the observation noise vector (of mean zero and covariance $\left.W^{-1}\right)$. $\tilde{D}$ is the geometric source function matrix between $x$ and $\tilde{b}$, of size $3 n \times 3 m$. In order to normalize $\tilde{\nu}$, we multiply (A14) by $W^{1 / 2}$ :

$$
b=D x+v
$$

[69] The elements of $D$ are given by equations (A11), (A12), and (A13). The inverse problem is solved by seeking the minimum of $L(x)=\nu^{T} \nu$, which corresponds to the normal equations:

$$
D^{T} D x=D^{T} b
$$

[70] When considering large problems, the computation of the product $D^{T} D$ can be very time consuming. It is then easier to use conjugate gradient approaches. Indeed the minimum for $L$ is reached when $\nabla L=D x-b$ goes to zero [Press et al., 1992]. We use an iterative process where we compute for each step $k$ a new solution $x_{k+1}$ equal to $x_{k}+$ $\alpha_{k} p_{k}$, where the vector $p_{k}$ is a search direction and $\alpha_{k}$ is a scalar minimizing $L\left(x_{k+1}\right)$ along $p_{k}$ :

$$
\alpha_{k}=\frac{r_{k}^{T} r_{k}}{p_{k}^{T} D^{T} D p_{k}}
$$

where $r_{k}$ is the vector of the residuals after the $k$ th iteration:

$$
r_{k}=D^{T} b-D^{T} D x_{k}
$$

[71] By using the matrix identity $p_{k}^{T} D^{T} D p_{k}=\left(D p_{k}\right)^{T} D p_{k}$ in equation (A17), we can use $D$ directly instead of having to calculate the product $D^{T} D$. This is called the design matrix approach [van der Sluis and van der Vorst, 1987].

\section{Appendix B: Influence of the Dipole Mesh}

[72] In an attempt to evaluate the influence of the position of each dipole on the resulting magnetization distribution, we performed several tests in which the input dipole meshes were rotated with respect to the original one we described in the paper. We used identical parameters $\left(i_{s}=23, h=-20\right)$. We considered three rotations, around the North Pole $\left(0^{\circ} \mathrm{E}\right.$, $\left.90^{\circ} \mathrm{N}\right)$, and around two equatorial locations, $\left(0^{\circ} \mathrm{E}, 0^{\circ} \mathrm{N}\right)$ and $\left(90^{\circ} \mathrm{E}, 0^{\circ} \mathrm{N}\right)$. These tests are referred as $\mathrm{R}_{\mathrm{Z}}, \mathrm{R}_{\mathrm{X}}$ and $\mathrm{R}_{\mathrm{Y}}$. In each case, the rotation angle is set to $1.5^{\circ}$, which corresponds to half the mean resolution of our input dipole mesh.

[73] First, the 1\% limit for the relative evolution of the residual rms values is reached after 16, 14 and 16 iterations for $R_{X}, R_{Y}$ and $R_{Z}$, respectively. The residual rms values and correlation coefficients we computed are similar to those of the M23/-20/14 model. Second, at 200-km altitude, rms differences between the M23/-20/14 model and the $\mathrm{R}_{\mathrm{X}}, \mathrm{R}_{\mathrm{Y}}$ and $\mathrm{R}_{\mathrm{Z}}$ models are as little as $3 \mathrm{nT}$, with correlation coefficients larger than 0.995. Finally, although the dipoles are not located at the same positions, we evaluated the rms differences and the correlation coefficients between the M23/-20/14 magnetization distribution 
and the $\mathrm{R}_{\mathrm{X}}, \mathrm{R}_{\mathrm{Y}}$ and $\mathrm{R}_{\mathrm{Z}}$ ones. Rms differences are of the order of $0.4 \mathrm{~A} / \mathrm{m}$, while correlation coefficients are larger than 0.9. These metrics were estimated using interpolated projections of the $R_{X}, R_{Y}$ and $R_{Z}$ models onto the M23/ $-20 / 14$ input dipole mesh.

[74] Although the location of the dipoles has a small impact on the final magnetization distribution, it is worth noting that these differences are very short length-scale, and do not affect the global characteristics of the magnetization distribution. Inclinations and declinations we estimated in Table 4 do not vary significantly. Paleopole positions based on the M23/-20/14 and on the three $R_{X}, R_{Y}$ and $R_{Z}$ tests fall within $10^{\circ}$ of each other for nine of the 10 anomalies described in Table 4. Similarly, the impact craters we described in Table 3 are still associated with weaker magnetized areas.

[75] Acknowledgments. B. Langlais is supported by a NAS/NRC postdoctoral fellowship. Authors would like to thank the MGS MAG-ER team and people of the Planetary Plasma Interactions Node of the Planetary Data System (PDS). We acknowledge Coerte Voorhies, who helped us on the conversion of our equivalent source model to a spherical harmonic one, Kathy Whaler, for helpful comments on the text, Terry Sabaka, for the covariance and error computation, and the editors and two anonymous referees. All graphs and maps have been plotted using the Generic Mapping Tools software [Wessel and Smith, 1991].

\section{References}

Acuña, M. H., et al. (1992), Mars Observer magnetic fields investigation, J. Geophys. Res, 97, 7799-7814.

Acuña, M. H., et al. (1998), Magnetic field and plasma observations at Mars: Initial results of the Mars Global Surveyor mission, Science, 279, 1676-1680.

Acuña, M. H., et al. (1999), Global distribution of crustal magnetization discovered by the Mars Global Surveyor MAG/ER experiment, Science, 284, 790-793.

Acuña, M. H., et al. (2001), Magnetic field of Mars: Summary of results from the aerobraking and mapping orbits, J. Geophys. Res., 106, 23,40323,417 .

Albee, A. L., D. Palluconi, and R. E. Arvidson (1998), Mars Global Surveyor Mission: Overview and status, Science, 279, 1671-1672.

Albee, A. L., R. E. Arvidson, F. Palluconi, and T. Thorpe (2001), Overview of the Mars Global Surveyor mission, J. Geophys. Res., 106, 23,29123,316.

Arkani-Hamed, J. (2001a), A 50-degree spherical harmonic model of the magnetic field of Mars, J. Geophys. Res., 106, 23,197-23,208.

Arkani-Hamed, J. (2001b), Paleomagnetic pole positions and pole reversals of Mars, Geophys. Res. Lett., 28, 3409-3412.

Arkani-Hamed, J. (2002), An improved 50-degree spherical harmonic model of the magnetic field of Mars derived from both high-altitude and low-altitude data, J. Geophys. Res., 107(E10), 5083, doi:10.1029/ 2001JE001835.

Arkani-Hamed, J., and D. Boutin (2003), Polar wander on Mars: Evidence from magnetic anomalies, in 6th International Conference on Mars, abstract 3051, Lunar and Planet. Inst., Houston, Tex.

Arkani-Hamed, J., R. A. Langel, and M. E. Purucker (1994), Scalar magnetic anomaly maps of Earth derived from POGO and MAGSAT data, J. Geophys. Res., 99, 24,075-24,090.

Cain, J. C., Z. Wang, C. Kluth, and D. R. Schmitz (1989), Derivation of geomagnetic model to $\mathrm{n}=63$, Geophys. J., 97, 431-441.

Cain, J. C., B. B. Ferguson, and D. Mozzoni (2003), An n $=90$ internal potential function of the Martian crustal magnetic field, J. Geophys. Res., 108(E2), 5008, doi:10.1029/2000JE001487.

Christensen, P. R., R. V. Morris, M. D. Lane, J. L. Bandfield, and M. C. Malin (2001), Global mapping of Martian hematite mineral deposits: Remnants of water-driven processes on early Mars, J. Geophys. Res., 106, 23,873-23,886.

Connerney, J. E. P., M. H. Acuña, P. J. Wasilewski, N. F. Ness, H. Rème, C. Mazelle, D. Vignes, R. P. Lin, D. Mitchell, and P. Cloutier (1999), Magnetic lineations in the ancient crust of Mars, Science, 284, 794798.

Covington, J. (1993), Improvement of equivalent source inversion technique with a more symmetric dipole distribution model, Phys. Earth Planet. Inter., 76, 199-208.
Dunlop, D. J., and G. Kletetschka (2001), Multidomain hematite: A source for planetary magnetic anomalies?, Geophys. Res. Lett., 28, 3345-3348. Dyment, J., and J. Arkani-Hamed (1998), Contribution of the lithospheric remanent magnetization to satellite magnetic anomalies over the world's oceans, J. Geophys. Res., 103, 15,423-15,441.

Garvin, J. B., S. E. H. Sakimoto, J. J. Frawley, and C. C. Schnetzler (2000), North polar region craterforms on Mars: Geometric characteristics from the Mars Orbiter Laser Altimeter, Icarus, 144, 329-352.

Gauss, C. F. (1839), Allgemeine Theorie des Erdmagnetismus, in Resultate aus den Beobachtungen Magnetischen Vereins im Jahre 1838, pp. 1-57, Weidmann, Leipzig, Germany. (Reprinted in Werke, 5, 121-193, 1877; translated by E. Sabine in Scientific Memoirs, vol. 2, edited by R. Taylor, pp. 184-251, Taylor and Taylor, London, 1841).

Halekas, J. S., and R. P. Lin (2003), Magnetic fields of lunar impact basins and their use in constraining the impact process, in 6th Workshop on Impact Cratering, abstract 8003, Lunar and Planet. Inst., Houston, Tex.

Halekas, J. S., D. L. Mitchell, R. P. Lin, L. L. Hood, M. H. Acuña, and A. B. Binder (2002), Demagnetization signatures of lunar impact craters, Geophys. Res. Lett., 29(13), 1645, doi:10.1029/2001GL013924.

Hood, L. L., N. C. Richmond, E. Pierazzo, and P. Rochette (2003), Distribution of crustal magnetic fields on Mars: Shock effects of basin-forming impacts, Geophys. Res. Lett., 30(6), 1281, doi:10.1029/ 2002GL016657.

Hynek, B. M., R. E. Arvidson, and R. J. Phillips (2002), Geologic setting and origin of Terra Meridiani hematite deposit on Mars, J. Geophys. Res., 107(E10), 5088, doi:10.1029/2002JE001891.

Kobayashi, H., M. Sato, T. Kamimura, M. Sakai, H. Onodera, N. Kuroda, and Y. Yamaguchi (1997), The effect of pressure on the electronic states of $\mathrm{FeS}$ and $\mathrm{Fe}_{7} \mathrm{~S}_{8}$ studied by Mossbauer spectroscopy, J. Phys. Condens. Matter, 9, 515-527.

Krymskii, A. M., T. K. Breus, N. F. Ness, M. H. Acuña, J. E. P. Connerney, D. H. Crider, D. L. Mitchell, and S. J. Bauer (2002), Structure of the magnetic field fluxes connected with crustal magnetization and topside ionosphere at Mars, J. Geophys. Res., 107(A9), 1245, doi:10.1029/ 2001JA000239.

Langel, R. A., and W. J. Hinze (1998), The magnetic field of the Earth's lithosphere: The satellite perspective, 429 pp., Cambridge Univ. Press, New York.

Langlais, B., M. Mandea, and P. Ultré-Guérard (2003), High-resolution magnetic field modeling: Application to MAGSAT and Ørsted data, Phys. Earth Planet. Inter., 135, 77-91.

Maus, S., M. Rother, R. Holme, H. Lühr, N. Olsen, and V. Haak (2002), First scalar magnetic anomaly map from CHAMP satellite data indicates weak lithospheric field, Geophys. Res. Lett., 29(14), 1702, doi:10.1029/ 2001 GL013685.

Mayhew, M. A. (1979), Inversion of satellite magnetic anomaly data, Geophys. J., 45, 119-128.

Mayhew, M. A., R. H. Estes, and D. M. Myers (1984), Remanent magnetization and three-dimensional density model of the Kentucky anomaly region, NASA Contract NAS5-27488, 90 pp.

Mitchell, D. L., R. P. Lin, H. Rme, P. A. Cloutier, J. E. P. Connerney, and N. F. Ness (2002), Probing Mars' crustal magnetic field and ionosphere with the MGS Electron Reflectometer, Lunar Planet. Sci., XXXIII, abstract 2029

Nimmo, F., and M. S. Gilmore (2001), Constraints on the depth of magnetized crust on Mars from impact craters, J. Geophys. Res., 106, 11,31511,323 .

Parker, R. L. (1977), Understanding inverse theory, Annu Rev. Earth Planet. Sci., 5, 35-64.

Parker, R. L. (2003), Ideal bodies for Mars magnetics, J. Geophys. Res., 108(E1), 5006, doi:10.1029/2001JE001760.

Pilkington, M., and R. A. F. Grieve (1992), The geophysical signature of terrestrial impact craters, Rev. Geophys., 30, 161-181.

Press, W. H., S. A. Teukolsky, W. T. Vetterling, and B. P. Flannery (1992), Numerical Recipes in C: The Art of Scientific Computing, 2nd ed., pp. 71-89, Cambridge Univ. Press, New York.

Purucker, M. E., T. J. Sabaka, and R. A. Langel (1996), Conjugate gradient analysis: A new tool for studying satellite magnetic datasets, Geophys. Res. Lett., 23, 507-510.

Purucker, M. E., D. Ravat, H. Frey, C. Voorhies, T. Sabaka, and M. Acuña (2000), An altitude-normalized magnetic map of Mars and its interpretation, Geophys. Res. Lett., 27, 2449-2452.

Ravat, D., K. A. Whaler, M. Pilkington, T. Sabaka, and M. Purucker (2002), Compatibility of high-altitude aeromagnetic and satellite-altitude magnetic anomalies over Canada, Geophysics, 67, 546-554.

Richmond, N. C., and L. L. Hood (2003), Paleomagnetic pole positions of Mars, Lunar Planet. Sci., XXXIII, abstract 1721.

Rochette, P., J. P. Lorand, G. Fillion, and V. Sautter (2001), Pyrrhotite and the remanent magnetization of SNC meteorites: A changing perspective on Martian magnetism, Earth Planet. Sci. Lett., 190, 1-12. 
Rochette, P., G. Fillion, R. Ballou, F. Brunet, B. Oulladiaf, and L. Hood (2003), High pressure magnetic transition in pyrrhotite and impact demagnetization on Mars, Geophys. Res. Lett., 30(13), 1683, doi:10.1029/ 2003GL017359.

Runcorn, S. K. (1975), On the interpretations of lunar magnetism, Phys. Earth Planet. Inter., 10, 327-335.

Sabaka, T. J., N. Olsen, and R. A. Langel (2002), A comprehensive model of the quiet-time, near-Earth magnetic field: Phase 3, Geophys. J. Int., $151,32-68$.

Schmitz, D. R., J. Meyer, and J. C. Cain (1989), Modeling the Earth's magnetic field to high degree and order, Geophys. J., 97, 421-430.

Smith, D. E., and M. T. Zuber (2002), The crustal thickness of Mars: Accuracy and resolution, Lunar Planet. Sci., XXXIII, abstract 1893.

Stevenson, D. J. (2001), Mars' core and magnetism, Nature, 412, 214 219.

Trotignon, J. G., R. Grard, and A. Skalsky (1993), Position and shape of the Martian bow shock: The Phobos2 plasma wave system observations, Planet. Space Sci., 41, 189-198.

van der Sluis, A., and H. A. van der Vorst (1987), Numerical solution of large, sparse linear algebraic systems arising from tomographic problems, in Seismic Tomography, edited by G. Nolet, pp. 49-83, D. Reidel, Norwell, Mass.

Vaughan, D. J., and J. A. Tossell (1973), Magnetic transitions observed in sulfide minerals at elevated pressures and their geophysical significance, Science, 179, 375-377.
Vennerstrom, S., N. Olsen, M. Purucker, M. H. Acuña, and J. C. Cain (2003), The magnetic field in the pile-up region at Mars, and its variation with the solar wind, Geophys. Res. Lett., 30(7), 1369, doi:10.1029/ 2003GL016883.

Vestine, E. H., W. L. Sibley, J. W. Kern, and J. L. Carlstedt (1963), Integral and spherical-harmonic analysis of the geomagnetic field for 1955.0, Part 2, J. Geomagn. Geoelectr., 15, 73-89.

Voorhies, C. V., T. J. Sabaka, and M. Purucker (2002), On magnetic spectra of Earth and Mars, J. Geophys. Res., 107(E6), 5034, doi:10.1029/ 2001JE001534.

Wessel, P., and W. H. F. Smith (1991), Free software helps map and display data, Eos Trans. AGU, 72, 441-448.

Whaler, K., and M. Purucker (2003), Martian magnetization-Preliminary models, Leading Edge, 22(8), 763-765.

Zuber, M. T. (2001), The crust and mantle of Mars, Nature, 412, 220-227.

B. Langlais, NAS/NRC at Geodynamics Branch, NASA Goddard Space Flight Center, Greenbelt, MD 20771, USA. (langlais@1tpmail.gsfc.nasa. gov)

M. Mandea, Institut de Physique du Globe, 4 Place Jussieu, 75252 Paris cedex 05, France. (mioara@ipgp.jussieu.fr)

M. E. Purucker, Raytheon ITSS at Geodynamics Branch, NASA Goddard Space Flight Center, Greenbelt, MD 20771, USA. (purucker@1tpmail.gsfc. nasa.gov) 